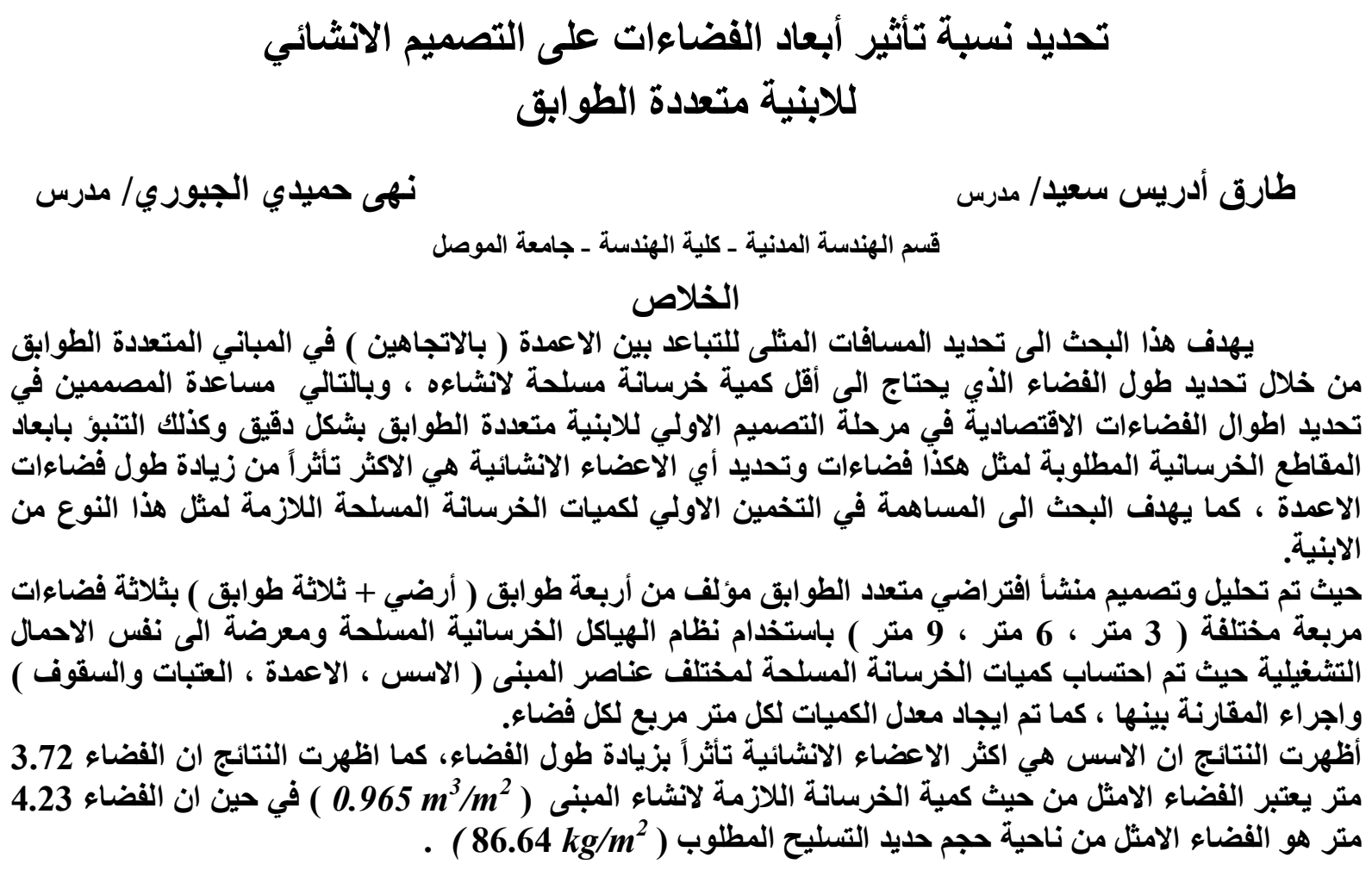

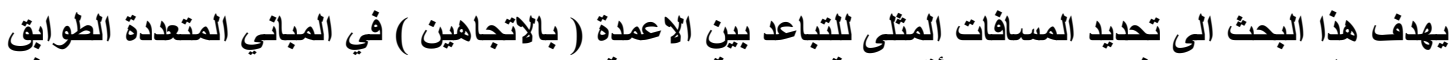

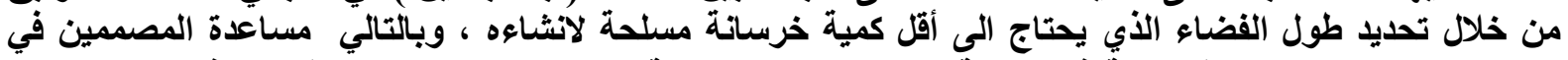

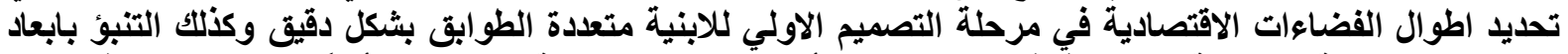

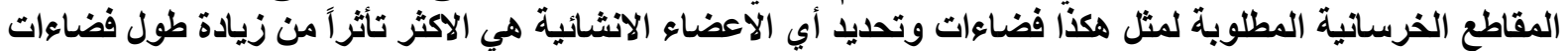

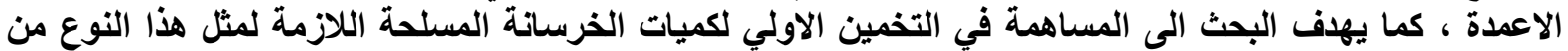

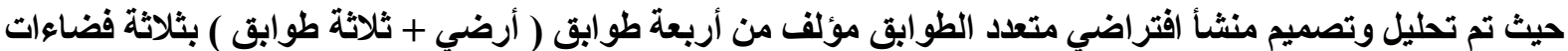

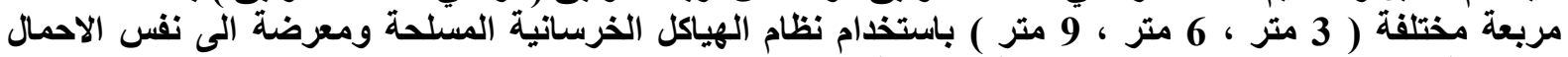

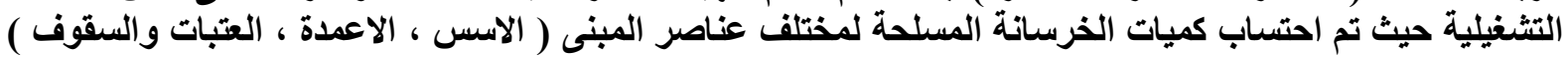

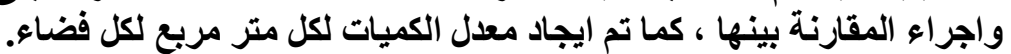

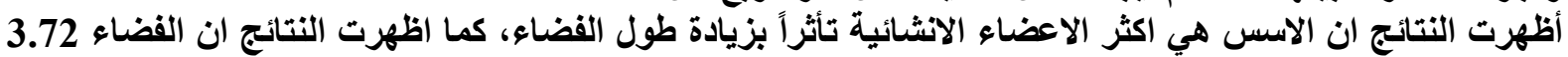

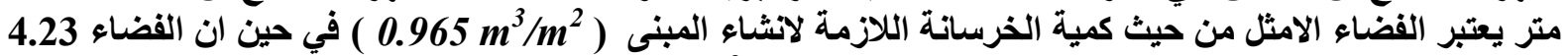

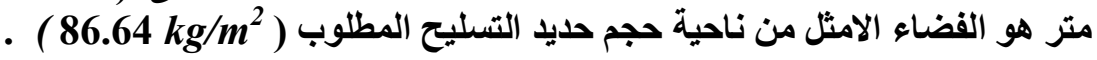

Key Words: Buildings Structural Design, Columns Spacing, Optimum Span, Preliminary Design, Skeleton Structures.

\title{
Effect of Spans Length on Structural Design of Multi story Buildings
}

\author{
Tarek Edrees Saaed / Lecturer Nuha Hemedy Al-Jubory/ Lecturer \\ College of Engg. Univ. of Mosul
}

\begin{abstract}
The aim of this research is to obtain the optimum spacing between columns in both directions for multi story buildings through the optimization of reinforced concrete required to construct them. This will help the designer in determining the economic spacing in the preliminary design stage, to predict the cross section sizes of members for these spans and to determine the most influenced structural members. A four story (ground +3 floors ) virtual reinforced concrete building was analyzed and designed for three cases of columns spacing $(3.0 \mathrm{~m}, 6.0 \mathrm{~m}, 9.0 \mathrm{~m})$ using reinforced concrete frame structures for the same working load conditions. A detailed estimation and comparison for reinforced concrete quantities was carried out for the structural members and the average per square meter was calculated.

The results showed that the foundation was the most influenced members due to increase in the span of columns, $3.72 \mathrm{~m}$ was the optimum spacing concerning the quantity of Concrete required for construction $\left(0.965 \mathrm{~m}^{3} / \mathrm{m}^{2}\right)$ while the span of $4.23 \mathrm{~m}$ was the optimum span for reinforcing steel $\left(86.64 \mathrm{~kg} / \mathrm{m}^{2}\right)$.
\end{abstract}




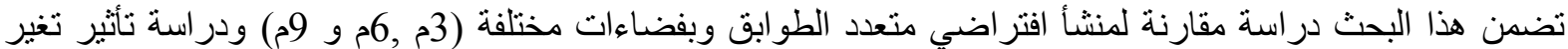

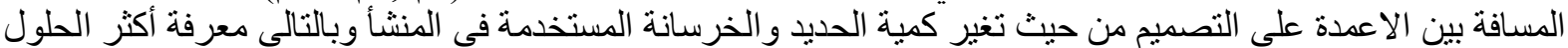

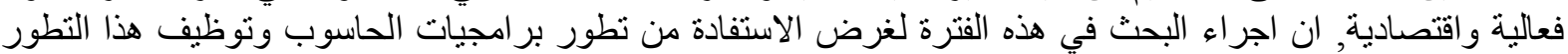
لحل بعض المشاكل الهندسية الجران

تحليل وتصميم المنشأ :

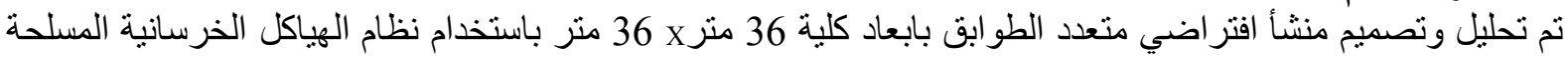

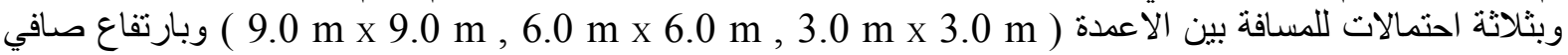
للبناية ( لا يضم عمق العتبات ) مساوي لـ 3.0 m للاحتمالات الثثلاثة وكما مبين في الثكل (1) باستخدام برنامج .STAAD.Pro 2007

تم تحميل المنشأ بحالات التحميل التالية [1]،[2] :

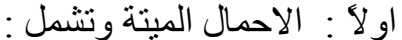

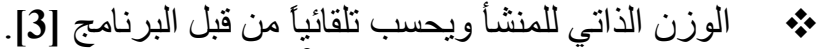

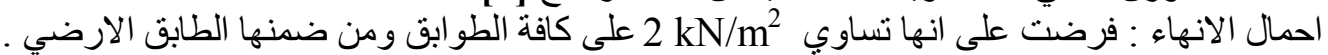

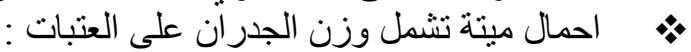

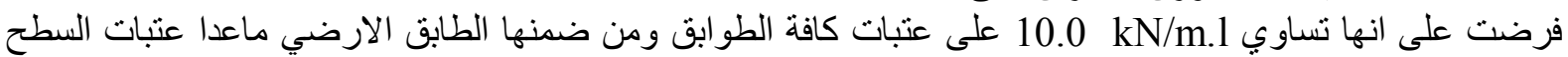

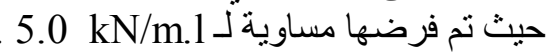

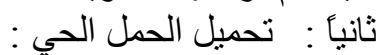
فرض على انه يساوي $2.5 \mathrm{kN} / \mathrm{m}^{2}$ فرياري

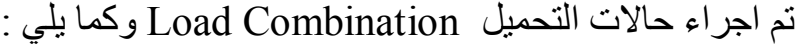
$\mathrm{U}=1.2$ D.L +1.6 L.L

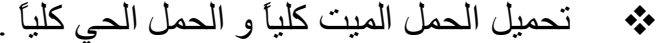
• تحميل الحمل الميت كلياً و الحمل الحي جزئياً وحسب نموذج رقعة الثطرنج ولجميع الطو ابق. $\mathrm{U}=1.2$ D.L +1.6 L. $\mathrm{L}^{(1,2)}$

ويبين الجدول (1) ادناه حالات التحميل المستخدمة في تحليل وتصميم المنشأ. جدول (1) : حالات التحميل المستخدمة في تحليل وتصميم المنشأ.

\begin{tabular}{|c|l|l|}
\hline & \multicolumn{1}{|c|}{ LOAD CASE NO. } & \multicolumn{1}{c|}{ LOAD TYPE } \\
\hline \hline 1. & Load 1 & Gravity \\
\hline 2. & Load 2 & Dead (F.L. + W.L.) \\
\hline 3. & Load 3 & Live (TOTAL L.L.) \\
\hline 4. & Load 4 & Live (L.L.1) - Checker Board Pattern \\
\hline 5. & Load 5 & Live (L.L.2) - Checker Board Pattern \\
\hline \hline & Load combination (Ultimate) & \\
\hline \hline 6. & Load combination 6 & $1 \times 1.2+2 \times 1.2+3 \times 1.6$ \\
\hline 7. & Load combination 7 & $1 \times 1.2+2 \times 1.2+4 \times 1.6$ \\
\hline 8. & Load combination 8 & $1 \times 1.2+2 \times 1.2+5 \times 1.6$ \\
\hline \hline & Load combination (Working) & \\
\hline \hline 9. & Load combination 9 & $1 \times 1.0+2 \times 1.0+3 \times 1.0$ \\
\hline 10. & Load combination 10 & $1 \times 1.0+2 \times 1.0+4 \times 1.0$ \\
\hline 11. & Load combination 11 & \\
\hline
\end{tabular}




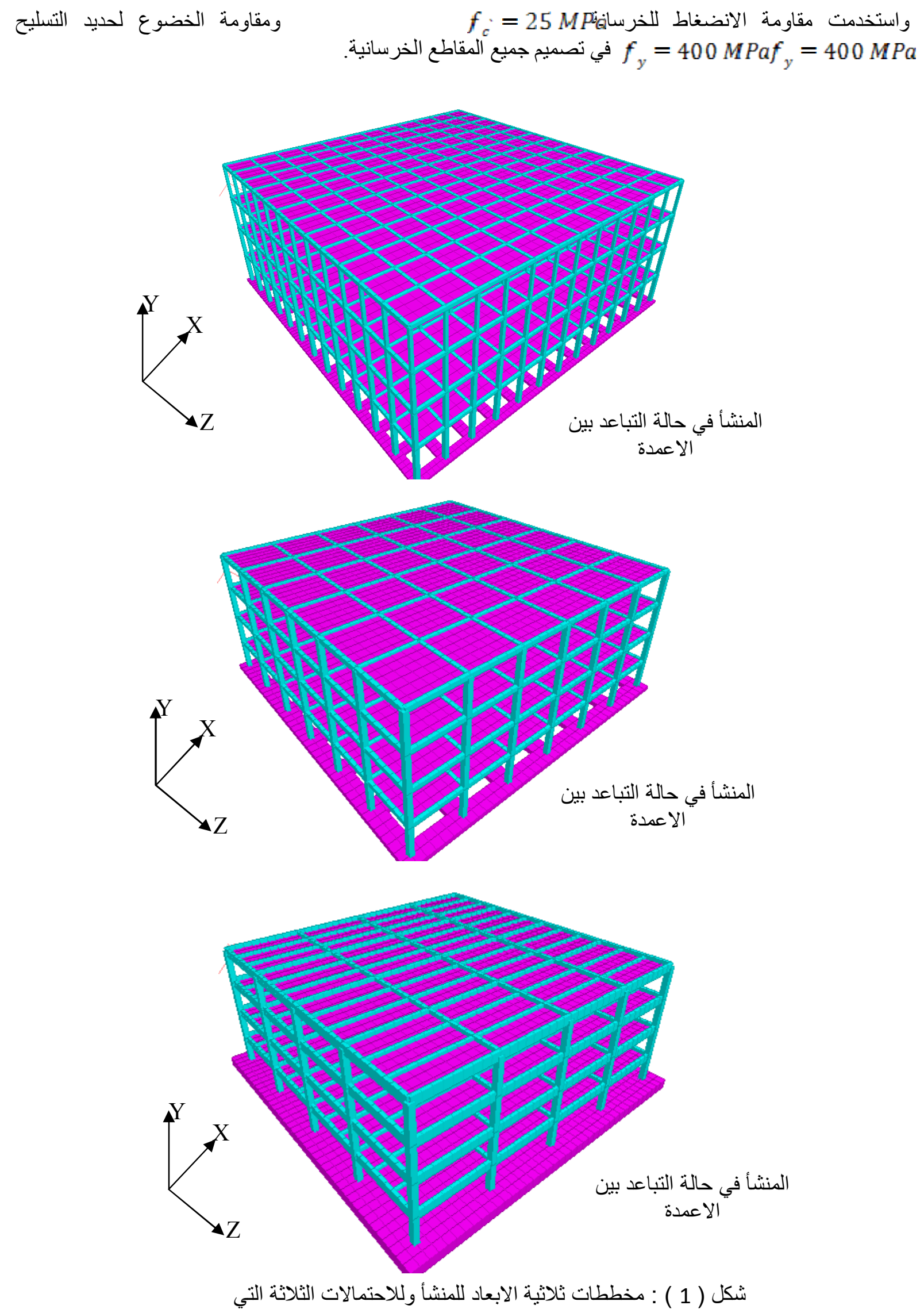




\section{نتائج التحليل و التصميم :}

تم تصميم المنشأ في الحالات الثلاثة عن طريق فرض قيم التيم لابعاد العتبات والاعمدة والاسس واعادة التصميم

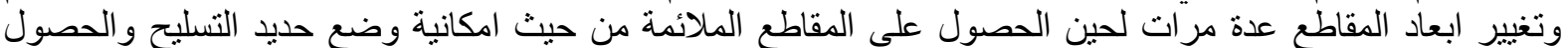

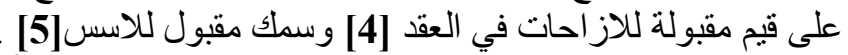

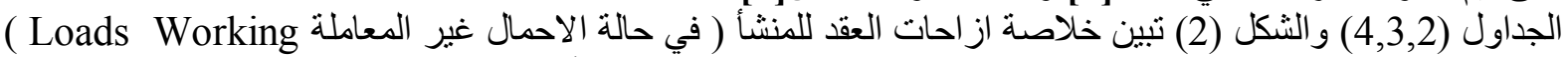

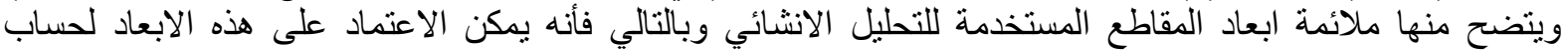
الكمبات التخمينية للخرسانة وحديد التنليح.

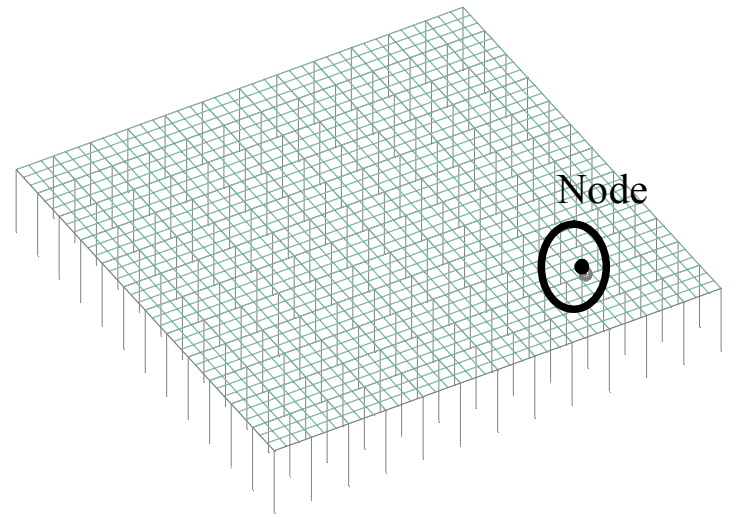

Columns Spacing $3 \mathrm{~m} \times 3 \mathrm{~m}$

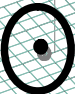

Node 4403 $\odot$

Node 3435

Columns Spacing $6 \mathrm{~m} \times 6 \mathrm{~m}$

Columns Spacing $9 \mathrm{~m} \times 9 \mathrm{~m}$

شكل (2) : مو اقع العقد التي حدثت فيها اقصى ازاحة بالاتجاه (Y) .

جدول (2) : خلاصة ازاحات العقد في حالة التباعد بين الاعمدة 3 m x 3 .

\begin{tabular}{|c|c|c|c|c|c|c|c|c|c|}
\hline & Node & $\mathrm{L} / \mathrm{C}$ & $\begin{array}{c}\mathrm{X} \\
(\mathrm{mm})\end{array}$ & $\begin{array}{c}\mathrm{Y} \\
(\mathrm{mm})\end{array}$ & $\begin{array}{c}\mathbf{Z} \\
(\mathrm{mm})\end{array}$ & $\begin{array}{l}\text { Resultant } \\
(\mathrm{mm})\end{array}$ & $\begin{array}{c}\mathrm{rX} \\
\text { (rad) }\end{array}$ & $\begin{array}{c}\mathrm{rY} \\
\text { (rad) }\end{array}$ & $\begin{array}{c}\mathrm{rZ} \\
\text { (rad) }\end{array}$ \\
\hline $\operatorname{Max} X$ & 806 & 11:COMBINAT & 0.016 & -4.839 & 0.016 & 4.839 & -0.000 & 0.000 & 0.000 \\
\hline $\operatorname{Min} X$ & 794 & 11:COMBINAT & -0.016 & -4.839 & 0.016 & 4.839 & -0.000 & -0.000 & -0.000 \\
\hline $\operatorname{Max} Y$ & 8336 & 11:COMBINAT & 0.000 & -3.772 & 0.000 & 3.772 & 0.001 & 0.000 & -0.000 \\
\hline $\operatorname{Min} Y$ & 1634 & 11:COMBINAT & -0.006 & -8.531 & -0.006 & 8.531 & -0.000 & 0.000 & 0.000 \\
\hline $\operatorname{Max} Z$ & 806 & 11:COMBINAT & 0.016 & -4.839 & 0.016 & 4.839 & -0.000 & 0.000 & 0.000 \\
\hline Min Z & 26 & 11:COMBINAT & 0.016 & -4.839 & -0.016 & 4.839 & 0.000 & 0.000 & 0.000 \\
\hline Max rX & 1170 & 11:COMBINAT & 0.004 & -6.538 & 0.009 & 6.538 & 0.001 & 0.000 & -0.000 \\
\hline Min $r X$ & 1752 & 11:COMBINAT & -0.004 & -6.538 & -0.009 & 6.538 & -0.001 & 0.000 & 0.000 \\
\hline Max rY & 25 & 11:COMBINAT & 0.011 & -5.312 & -0.015 & 5.312 & 0.000 & 0.000 & 0.000 \\
\hline Min $r Y$ & 729 & 11:COMBINAT & -0.015 & -5.312 & 0.011 & 5.312 & -0.000 & -0.000 & -0.000 \\
\hline Max rZ & 1578 & 11:COMBINAT & -0.009 & -6.538 & -0.004 & 6.538 & -0.000 & -0.000 & 0.001 \\
\hline Min $r Z$ & 1889 & 11:COMBINAT & 0.009 & -6.538 & -0.004 & 6.538 & -0.000 & 0.000 & -0.001 \\
\hline Max Rst & 1634 & 11:COMBINAT & -0.006 & -8.531 & -0.006 & 8.531 & -0.000 & 0.000 & 0.000 \\
\hline
\end{tabular}


سعيد: تحديد نسبة تأثير أبعاد الفضاعات على التصميم الانشائي للابنية متعددة الطوابق

جدول (3) : خلاصة ازاحات العقد في حالة التباعد بين الاعمدة 6 m 6 .

\begin{tabular}{|c|c|c|c|c|c|c|c|c|c|}
\hline & Node & $\mathrm{L} / \mathrm{C}$ & $\begin{array}{c}\mathrm{X} \\
(\mathrm{mm})\end{array}$ & $\begin{array}{c}\mathrm{Y} \\
(\mathrm{mm})\end{array}$ & $\begin{array}{c}\mathbf{Z} \\
(\mathrm{mm})\end{array}$ & $\begin{array}{l}\text { Resultant } \\
\text { (mm) }\end{array}$ & $\begin{array}{c}\mathrm{rX} \\
\text { (rad) }\end{array}$ & $\begin{array}{c}\mathrm{rY} \\
\text { (rad) }\end{array}$ & $\begin{array}{c}\mathrm{rZ} \\
\text { (rad) }\end{array}$ \\
\hline $\operatorname{Max} X$ & 204 & 10:COMBINAT & 0.053 & -8.168 & 0.030 & 8.169 & 0.000 & 0.000 & -0.001 \\
\hline $\operatorname{Min} X$ & 238 & 10:COMBINAT & -0.053 & -8.168 & -0.030 & 8.169 & -0.000 & 0.000 & 0.001 \\
\hline $\operatorname{Max} Y$ & 6630 & 11:COMBINAT & 0.000 & -3.256 & 0.000 & 3.256 & -0.000 & 0.000 & -0.000 \\
\hline $\operatorname{Min} Y$ & 3435 & 10:COMBINAT & -0.001 & -16.023 & -0.001 & 16.023 & -0.000 & 0.000 & 0.000 \\
\hline $\operatorname{Max} Z$ & 198 & 10:COMBINAT & 0.030 & -8.168 & 0.053 & 8.169 & 0.001 & -0.000 & -0.000 \\
\hline Min Z & 244 & 10:COMBINAT & -0.030 & -8.168 & -0.053 & 8.169 & -0.001 & -0.000 & 0.000 \\
\hline $\operatorname{Max} r X$ & 3056 & 10:COMBINAT & 0.004 & -11.714 & -0.007 & 11.714 & 0.002 & 0.000 & 0.000 \\
\hline $\operatorname{Min} r X$ & 4162 & 10:COMBINAT & 0.004 & -11.714 & 0.007 & 11.714 & -0.002 & -0.000 & 0.000 \\
\hline Max rY & 4251 & 10:COMBINAT & 0.026 & -8.518 & 0.042 & 8.518 & 0.001 & 0.000 & -0.000 \\
\hline Min $r Y$ & 4452 & 10:COMBINAT & 0.042 & -8.518 & 0.026 & 8.518 & 0.000 & -0.000 & -0.001 \\
\hline Max $r Z$ & 3972 & 10:COMBINAT & 0.007 & -11.714 & 0.004 & 11.714 & -0.000 & 0.000 & 0.002 \\
\hline Min rZ & 3790 & 10:COMBINAT & -0.007 & -11.714 & 0.004 & 11.714 & -0.000 & -0.000 & -0.002 \\
\hline Max Rst & 3435 & 10:COMBINAT & -0.001 & -16.023 & -0.001 & 16.023 & -0.000 & 0.000 & 0.000 \\
\hline
\end{tabular}

جدول (4) : خلاصة ازاحات العقد في حالة التباعد بين الاعمدة m x 9 m

\begin{tabular}{|c|c|c|c|c|c|c|c|c|c|}
\hline & Node & $\mathrm{L} / \mathrm{C}$ & $\begin{array}{c}\mathrm{X} \\
(\mathrm{mm})\end{array}$ & $\begin{array}{c}\mathrm{Y} \\
(\mathrm{mm})\end{array}$ & $\begin{array}{c}\mathbf{Z} \\
(\mathrm{mm})\end{array}$ & $\begin{array}{c}\text { Resultant } \\
\text { (mm) }\end{array}$ & $\begin{array}{c}\mathrm{rX} \\
(\mathrm{rad})\end{array}$ & $\begin{array}{c}\mathrm{rY} \\
(\mathrm{rad})\end{array}$ & $\begin{array}{c}\mathrm{rZ} \\
(\mathrm{rad})\end{array}$ \\
\hline $\operatorname{Max} X$ & 4138 & 10:COMBINAT & 0.079 & -8.783 & 0.050 & 8.784 & -0.000 & -0.000 & -0.001 \\
\hline $\operatorname{Min} X$ & 4152 & 10:COMBINAT & -0.079 & -8.783 & -0.050 & 8.784 & 0.000 & -0.000 & 0.001 \\
\hline $\operatorname{Max} Y$ & 5641 & 12:COMBINAT & 0.000 & -3.259 & 0.000 & 3.259 & -0.000 & 0.000 & -0.000 \\
\hline $\operatorname{Min} Y$ & 4403 & 10:COMBINAT & -0.015 & -20.126 & 0.078 & 20.126 & -0.000 & -0.000 & 0.000 \\
\hline $\operatorname{Max} Z$ & 4134 & 10:COMBINAT & 0.030 & -9.040 & 0.151 & 9.042 & 0.002 & -0.000 & -0.000 \\
\hline $\operatorname{Min} Z$ & 4154 & 10:COMBINAT & 0.030 & -9.040 & -0.151 & 9.042 & -0.002 & 0.000 & -0.000 \\
\hline Max rX & 4208 & 10:COMBINAT & 0.042 & -12.992 & 0.085 & 12.992 & 0.004 & -0.000 & -0.000 \\
\hline $\operatorname{Min} r X$ & 5411 & 10:COMBINAT & 0.042 & -12.992 & -0.085 & 12.992 & -0.004 & 0.000 & -0.000 \\
\hline Max rY & 4302 & 10:COMBINAT & -0.036 & -9.210 & 0.118 & 9.211 & 0.002 & 0.000 & -0.000 \\
\hline Min $r Y$ & 5494 & 10:COMBINAT & -0.036 & -9.210 & -0.118 & 9.211 & -0.002 & -0.000 & -0.000 \\
\hline Max rZ & 3372 & 10:COMBINAT & 0.007 & -13.557 & -0.004 & 13.557 & 0.000 & -0.000 & 0.003 \\
\hline Min rZ & 3285 & 10:COMBINAT & -0.007 & -13.557 & -0.004 & 13.557 & 0.000 & 0.000 & -0.003 \\
\hline Max Rst & 4403 & 10:COMBINAT & -0.015 & -20.126 & 0.078 & 20.126 & -0.000 & -0.000 & 0.000 \\
\hline
\end{tabular}

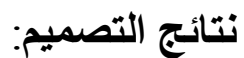

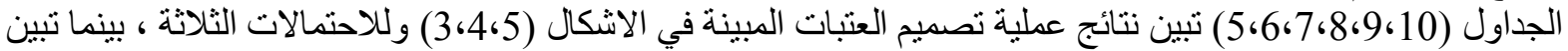

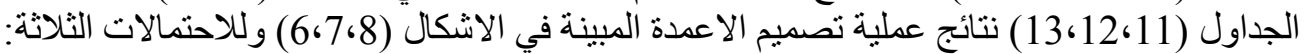

جدول (5) نتائج تصميم السقوف لفضاء (3×3) م

\begin{tabular}{|c|c|c|c|c|}
\hline الطابق & As + mm2 & As - mm2 & Asmin & As \\
\hline الطوابق & 159 & 129 & 180 & $\Phi 10 @ 300 \mathrm{mmc} / \mathrm{c}$ \\
\hline السطخ & 151 & 129 & 180 & $\Phi 10 @ 300 \mathrm{~mm} \mathrm{c/c}$ \\
\hline
\end{tabular}


جدول (6) نتائج تصميم السقوف لفضاء (6×6) م

\begin{tabular}{|c|c|c|c|c|}
\hline الطابق & As + mm2 & As - mm2 & As min & As \\
\hline المستخدمة & 355 & 338 & 270 & $\Phi 12 @ 300 \mathrm{mmc} / \mathrm{c}$ \\
\hline السطح & 266 & 203 & 270 & $\Phi 12 @ 400 \mathrm{~mm} \mathrm{c/c}$ \\
\hline
\end{tabular}

جدول (7) نتائج تصميم السقوف لفضاء (9×9) م

\begin{tabular}{|c|c|c|c|}
\hline & الاتجاه الطويل & As min \\
\hline الطاتجاه القصير & $\Phi 12 @ 350 \mathrm{~mm}$ & $\Phi 12 @ 225 \mathrm{mmc} / \mathrm{c}$ & 225 \\
\hline الطبق & $\Phi 12 @ 350 \mathrm{~mm}$ & $\Phi 12 @ 125 \mathrm{mmc} / \mathrm{c}$ & 225 \\
\hline
\end{tabular}

جدول (8) نتائج تصميم العتبات لفضاء (3×3) م

\begin{tabular}{|c|c|c|c|c|c|c|}
\hline الطابق & العتبة & $\begin{array}{c}\text { الابعاد } \\
\text { m }\end{array}$ & As - & As + & As min & Stirrups \\
\hline كل الطو ابق & B1 & $0.25 * 0.35$ & $3 \Phi 12$ & $3 \Phi 12$ & $3 \Phi 12 \mathrm{~mm}$ & $\Phi 10 @ 150 \mathrm{~mm} \mathrm{c} / \mathrm{c}$ \\
\hline كل الطو ابق & $\bar{B} 2$ & $0.25 * 0.35$ & $3 \Phi 12$ & $3 \Phi 12$ & $3 \Phi 12 \mathrm{~mm}$ & $\Phi 10 @ 150 \mathrm{~mm} \mathrm{c} / \mathrm{c}$ \\
\hline
\end{tabular}

جدول (9) نتائج تصميم العتبات لفضاء (6) (6) م

\begin{tabular}{|c|c|c|c|c|c|c|}
\hline الطابق & العتبة & الابعاد & As - & As + & As min & Stirrups \\
\hline السطح & B1 & $0.35 * 0.5$ & $5 \Phi 12$ & $4 \Phi 12$ & $5 \Phi 10 \mathrm{~mm}$ & $\Phi 10 @ 200 \mathrm{~mm} \mathrm{c} / \mathrm{c}$ \\
\hline السطح & B2 & $0.35 * 0.5$ & $5 \Phi 16$ & $4 \Phi 16$ & $5 \Phi 10 \mathrm{~mm}$ & $\Phi 10 @ 200 \mathrm{~mm} \mathrm{c} / \mathrm{c}$ \\
\hline باقي الطوابق & B1 & $0.35 * 0.5$ & $5 \Phi 16$ & $3 \Phi 16$ & $5 \Phi 10 \mathrm{~mm}$ & $\Phi 10 @ 200 \mathrm{~mm} \mathrm{c} / \mathrm{c}$ \\
\hline باقي الطو ابق & $\mathrm{B} 2$ & $0.35 * 0.5$ & $5 \Phi 20$ & $3 \Phi 20$ & $5 \Phi 10 \mathrm{~mm}$ & $\Phi 10 @ 200 \mathrm{~mm} \mathrm{c} / \mathrm{c}$ \\
\hline
\end{tabular}

جدول (10) نتائج تصميم العتبات لفضاء (9×9) م

\begin{tabular}{|c|c|c|c|c|c|c|}
\hline الطابق & العتبة & الابعاد & As - & Ast & As min & Stirrups \\
\hline \multirow[t]{5}{*}{ السطح } & B1 & $0.35 * 1.0$ & $3 \Phi 20$ & $3 \Phi 20$ & $6 \Phi 16 \mathrm{~mm}$ & $\Phi 10 @ 300 \mathrm{~mm}$ \\
\hline & B2 & $0.35 * 1.0$ & $6 \Phi 20$ & $5 \Phi 20$ & $6 \Phi 16 \mathrm{~mm}$ & $\Phi 10 @ 300 \mathrm{~mm}$ \\
\hline & B3 & $0.35 * 1.0$ & $5 \Phi 20$ & $7 \Phi 20$ & $6 \Phi 16 \mathrm{~mm}$ & Ф10@300 mm \\
\hline & B4 & $0.35 * 1.0$ & $6 \Phi 20$ & $5 \Phi 20$ & $6 \Phi 16 \mathrm{~mm}$ & $\Phi 10 @ 300 \mathrm{~mm}$ \\
\hline & B5 & $0.35 * 0.75$ & $5 \Phi 20$ & $4 \Phi 20$ & $5 \Phi 16 \mathrm{~mm}$ & $\Phi 10 @ 300 \mathrm{~mm}$ \\
\hline \multirow[t]{5}{*}{ باقي الطوابق } & B1 & $0.35 * 1.0$ & $3 \Phi 20$ & $6 \Phi 20$ & $6 \Phi 16 \mathrm{~mm}$ & $\Phi 10 @ 300 \mathrm{~mm}$ \\
\hline & $\mathrm{B} 2$ & $0.35 * 1.0$ & $6 \Phi 20$ & $5 \Phi 25$ & $6 \Phi 16 \mathrm{~mm}$ & $\Phi 10 @ 300 \mathrm{~mm}$ \\
\hline & B3 & $0.35 * 1.0$ & $5 \Phi 20$ & $7 \Phi 25$ & $6 \Phi 16 \mathrm{~mm}$ & $\Phi 10 @ 300 \mathrm{~mm}$ \\
\hline & B4 & $0.35 * 1.0$ & $6 \Phi 20$ & $6 \Phi 20$ & $6 \Phi 16 \mathrm{~mm}$ & $\Phi 10 @ 300 \mathrm{~mm}$ \\
\hline & B5 & $0.35 * 0.75$ & $5 \Phi 20$ & $6 \Phi 20$ & $5 \Phi 16 \mathrm{~mm}$ & $\Phi 10 @ 300 \mathrm{~mm}$ \\
\hline
\end{tabular}


سعيد: تحديد نسبة تأثير أبعاد الفضاءات على التصميم الانشائي للابنية متعددة الطوابق
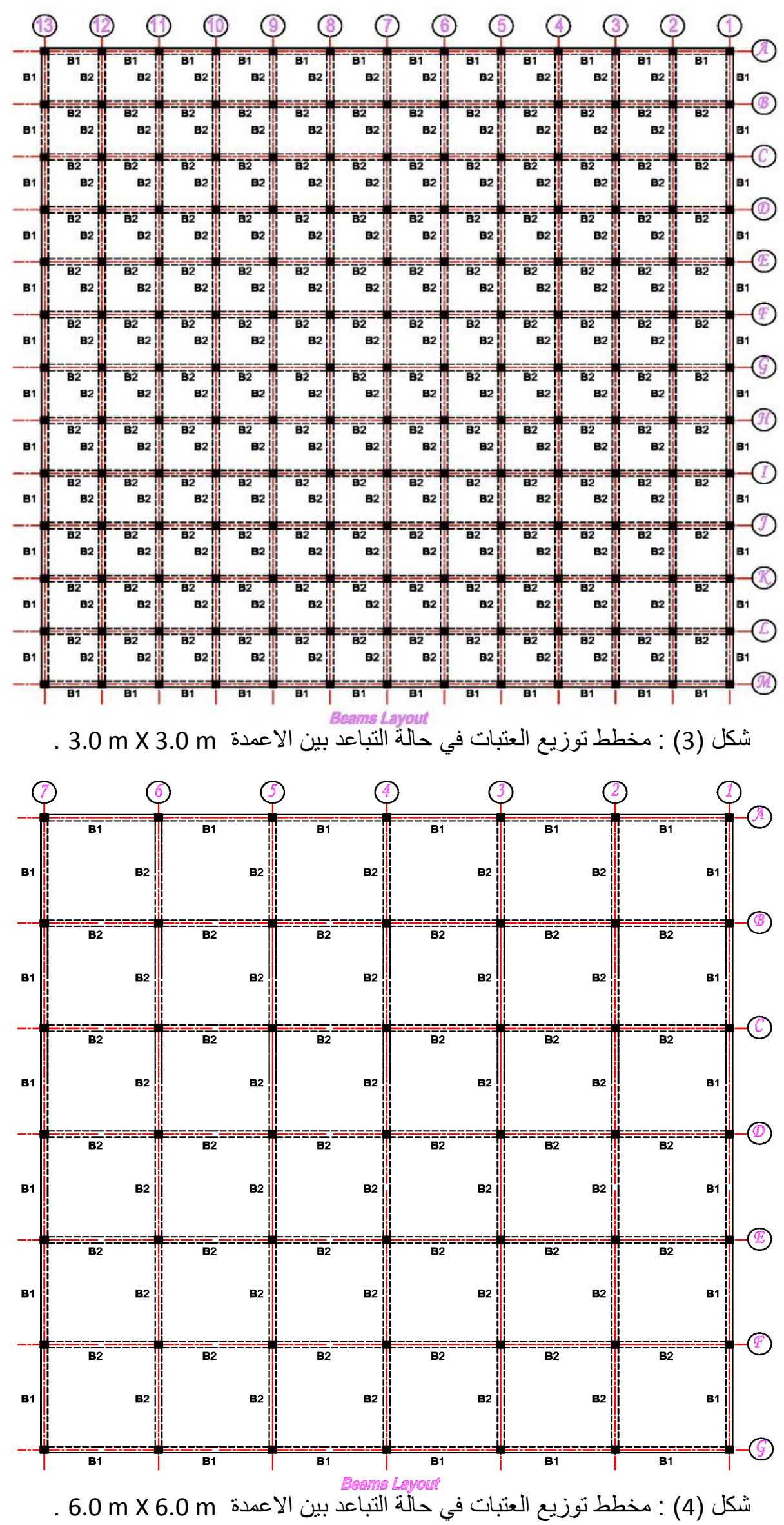


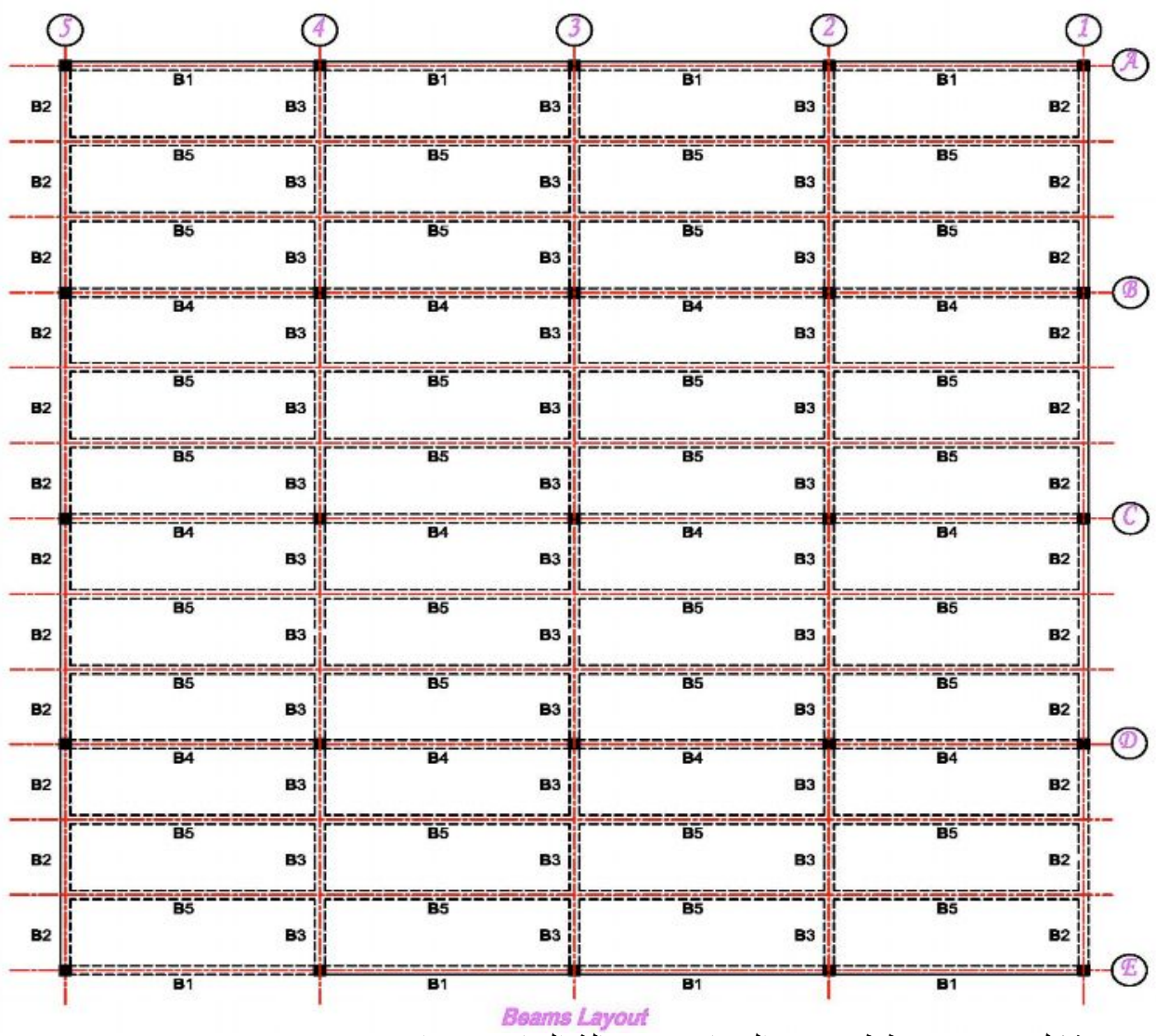

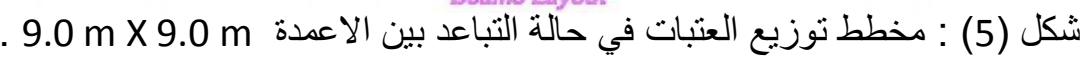

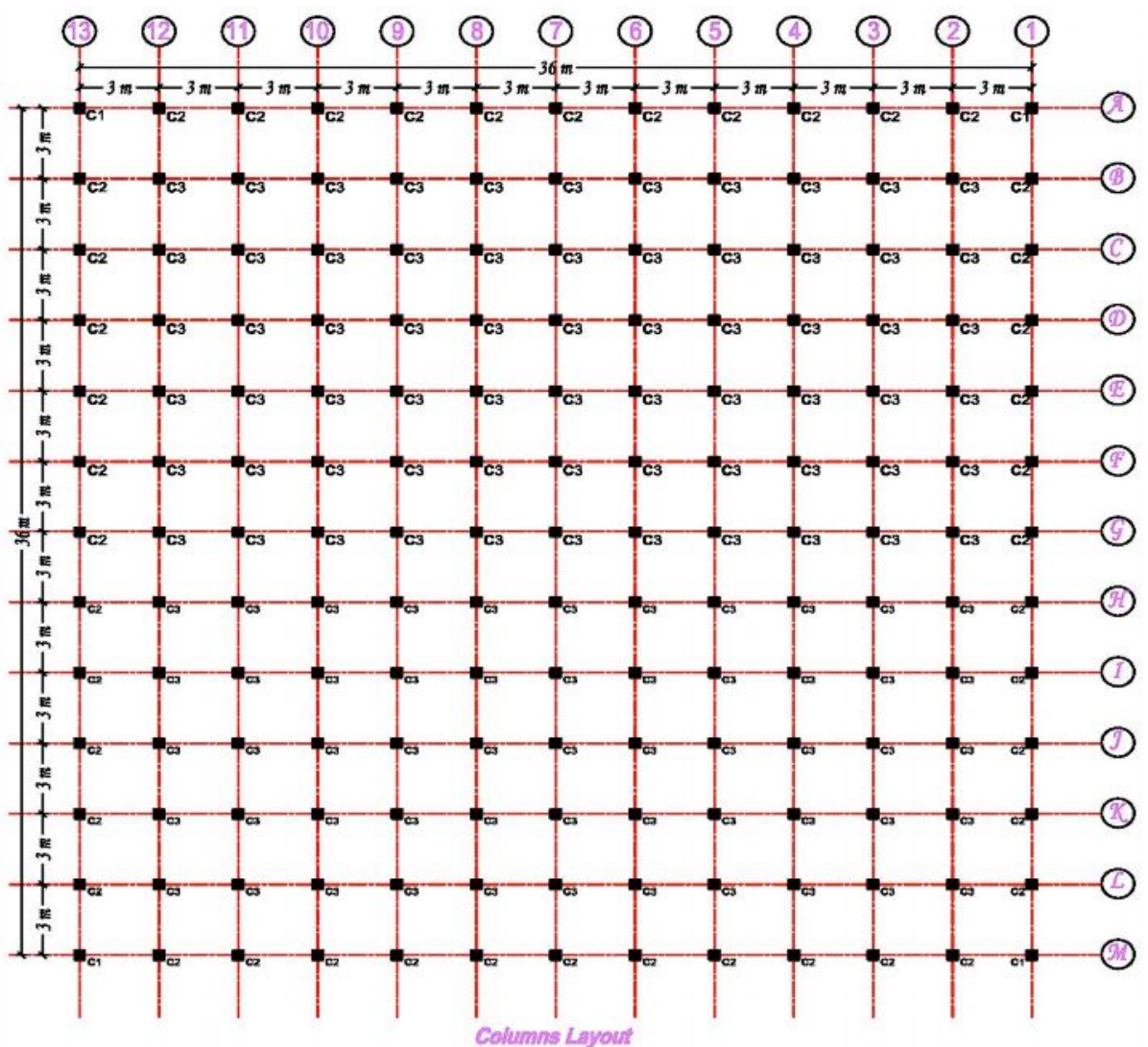

شكل (6) : مخطط توزيع الاعمدة في حالة التباعد بين الاعمدة 3 X 3.0 . 
سعيد: تحديد نسبة تأثير أبعاد الفضاءات على التصميم الانثائي للابنية متعددة الطوابق
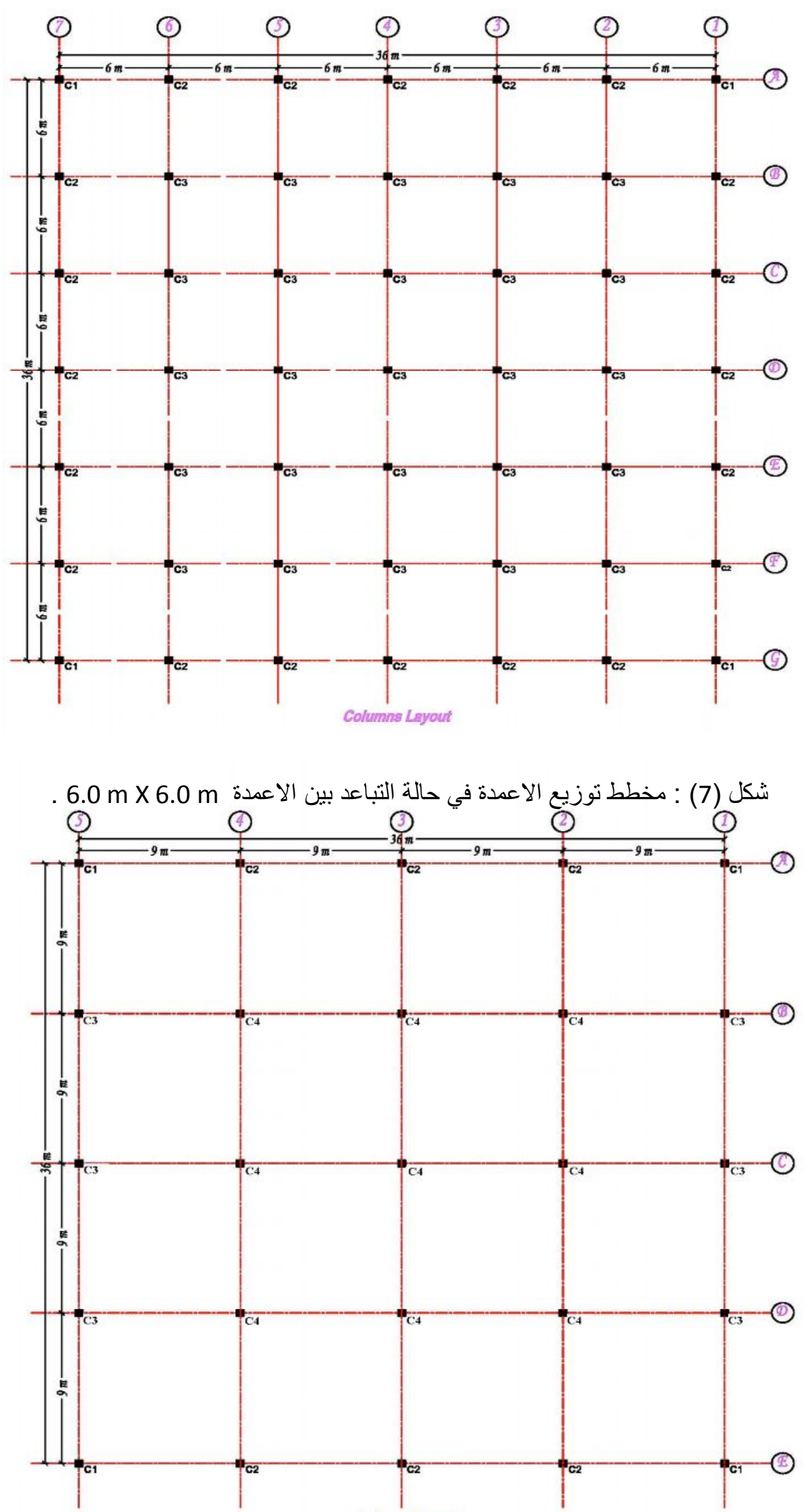

شكل (8) : مخطط توزيع الاعمدة في حألة ألتباعد بين الاعمدة 9.0 m X 9.0 m . 
No.4

August 2011

جدول (11) نتائج تصميم الاعمدة لفضاء (3×3) م إعباد العبداء

\begin{tabular}{|c|c|c|c|c|}
\hline الطابق & العمود & ابعاد العمود & As & Ties \\
\hline الارضي & $\mathrm{C} 1$ & $0.4 * 0.4$ & $4 \Phi 25 \mathrm{~mm}$ & $(1-\operatorname{set} \Phi 10 @ 400)$ \\
\hline الارضي & $\mathrm{C} 2$ & $0.4 * 0.4$ & $4 \Phi 25 \mathrm{~mm}$ & (1-set $\Phi 10 @ 400)$ \\
\hline الارضي & $\mathrm{C} 3$ & $0.4 * 0.4$ & $4 \Phi 25 \mathrm{~mm}$ & (1-set $\Phi 10 @ 400)$ \\
\hline الاول & $\mathrm{C} 1$ & $0.35 * 0.35$ & $4 \Phi 20 \mathrm{~mm}$ & (1-set $\Phi 10 @ 300)$ \\
\hline الاول & $\mathrm{C} 2$ & $0.35 * 0.35$ & $4 \Phi 20 \mathrm{~mm}$ & $(1-\operatorname{set} \Phi 10 @ 300)$ \\
\hline الاول & $\mathrm{C} 3$ & $0.35 * 0.35$ & $4 \Phi 20 \mathrm{~mm}$ & $(1-\operatorname{set} \Phi 10 @ 300)$ \\
\hline الثاني & $\mathrm{C} 1$ & $0.3 * 0.3$ & $4 \Phi 18 \mathrm{~mm}$ & (1-set $\Phi 10 @ 250)$ \\
\hline الثاني & $\mathrm{C} 2$ & $0.3 * 0.3$ & $4 \Phi 18 \mathrm{~mm}$ & (1-set $\Phi 10 @ 250)$ \\
\hline الثاني & $\mathrm{C} 3$ & $0.3 * 0.3$ & $4 \Phi 18 \mathrm{~mm}$ & (1-set $\Phi 10 @ 250)$ \\
\hline الثالث & $\mathrm{C} 1$ & $0.25 * 0.25$ & $4 \Phi 16 \mathrm{~mm}$ & (1-set $\Phi 10 @ 250)$ \\
\hline الثالث & $\mathrm{C} 2$ & $0.25 * 0.25$ & $4 \Phi 16 \mathrm{~mm}$ & $(1-\operatorname{set} \Phi 10 @ 250)$ \\
\hline الثالث & $\mathrm{C} 3$ & $0.25 * 0.25$ & $4 \Phi 16 \mathrm{~mm}$ & (1-set $\Phi 10 @ 250)$ \\
\hline
\end{tabular}

جدول (12) نتائج تصميم الاعمدة لفضاء (6×6) م

\begin{tabular}{|c|c|c|c|c|}
\hline الطابق & العمود & ابعاد العمود & As & Ties \\
\hline كل الطو ابق & $\mathrm{C} 1$ & $0.5 * 0.5$ & $4 \Phi 25+4 \Phi 20 \mathrm{~mm}$ & $(2-\operatorname{set} \Phi 10 @ 300)$ \\
\hline & $\mathrm{C} 2$ & $0.5 * 0.5$ & $8 \Phi 20 \mathrm{~mm}$ & $(2-\operatorname{set} \Phi 10 @ 300)$ \\
\hline & C3 & $0.5 * 0.5$ & $4 \Phi 25 \mathrm{~mm}$ & (2-set $\Phi 10 @ 300)$ \\
\hline
\end{tabular}

جدول (13) نتائج تصميم الاعمدة لفضاء (9×9) م

\begin{tabular}{|c|c|c|c|c|}
\hline الطابق & العمود & ابعاد العمود & As & Ties \\
\hline كل الطو ابق & $\mathrm{C} 1$ & $0.6 * 0.6$ & $12 \Phi 25 \mathrm{~mm}$ & (3-set $\Phi 10 @ 400)$ \\
\hline كل الطو ابق & $\mathrm{C} 2$ & $0.6 * 0.6$ & $20 \Phi 28 \mathrm{~mm}$ & (3-set $\Phi 10 @ 400)$ \\
\hline كل الطو ابق & $\mathrm{C} 3$ & $0.6 * 0.6$ & $16 \Phi 20 \mathrm{~mm}$ & (3-set $\Phi 10 @ 400)$ \\
\hline الارضي & $\mathrm{C} 4$ & $0.6 * 0.6$ & $28 \Phi 32 \mathrm{~mm}$ & (3-set $\Phi 10 @ 400)$ \\
\hline الاول & $\mathrm{C} 4$ & $0.6 * 0.6$ & $16 \Phi 28 \mathrm{~mm}$ & (3-set $\Phi 10 @ 400)$ \\
\hline الثاني & $\mathrm{C} 4$ & $0.6 * 0.6$ & $12 \Phi 20 \mathrm{~mm}$ & (3-set $\Phi 10 @ 400)$ \\
\hline الثالث & $\mathrm{C} 4$ & $0.6 * 0.6$ & $12 \Phi 20 \mathrm{~mm}$ & (3-set $\Phi 10 @ 400)$ \\
\hline
\end{tabular}

جدول (14) نتائج تصميم الاساس "لفضاء (3×3) م

\begin{tabular}{|c|c|c|}
\hline & As & As min \\
\hline طوليج| & $12 \Phi 16 \mathrm{~mm}$ & 1400 \\
\hline حلقيات & $\Phi 16 @ 250 \mathrm{~mm} \mathrm{c} / \mathrm{c}$ & 1400 \\
\hline
\end{tabular}

* نوع الاساس هو اساس مستمر.

جدول (15) نتائج تصميم الاساس* لفضاء (6) (6) م

\begin{tabular}{|c|c|c|}
\hline & As & As min \\
\hline طوع التسليح & $28 \Phi 20 \mathrm{~mm}$ & 1925 \\
\hline طلقات & $\Phi 20 @ 250 \mathrm{~mm} \mathrm{c} / \mathrm{c}$ & 1925 \\
\hline
\end{tabular}


سعيد: تحديد نسبة تأثير أبعاد الفضاءات على التصميم الانثائي للابنية متعددة الطوابق

\begin{tabular}{|c|c|c|}
\hline \multicolumn{3}{|c|}{ جدول (16) نتائج تصميم الاساس* لفضاء (9×9) م } \\
\hline نوع التسليح & As & As min \\
\hline شبكة اسفل و اعلى & $\Phi 28 @ 150 \mathrm{~mm} \mathrm{c} / \mathrm{c}$ & 2160 \\
\hline شبكة وسطي & Ф 28 (C) $300 \mathrm{~mm} \mathrm{c} / \mathrm{c}$ & 2160 \\
\hline
\end{tabular}

* نو ع الاساس هو اساس حصيري ونلاحظ الحاجة الى شبكة تسليح اضافية ( وسطى ) بسبب زيادة سمك الاساس عن 1.0 منر مما تسبب في زيل زيادة أضافية لحديد التسليح.

تبين الجداول (16،15،14) نتائج عملية تصميم الاسس المبينة في الاشكال (14،13،12،11،10،9) وللاحتمالات الثلاثة:

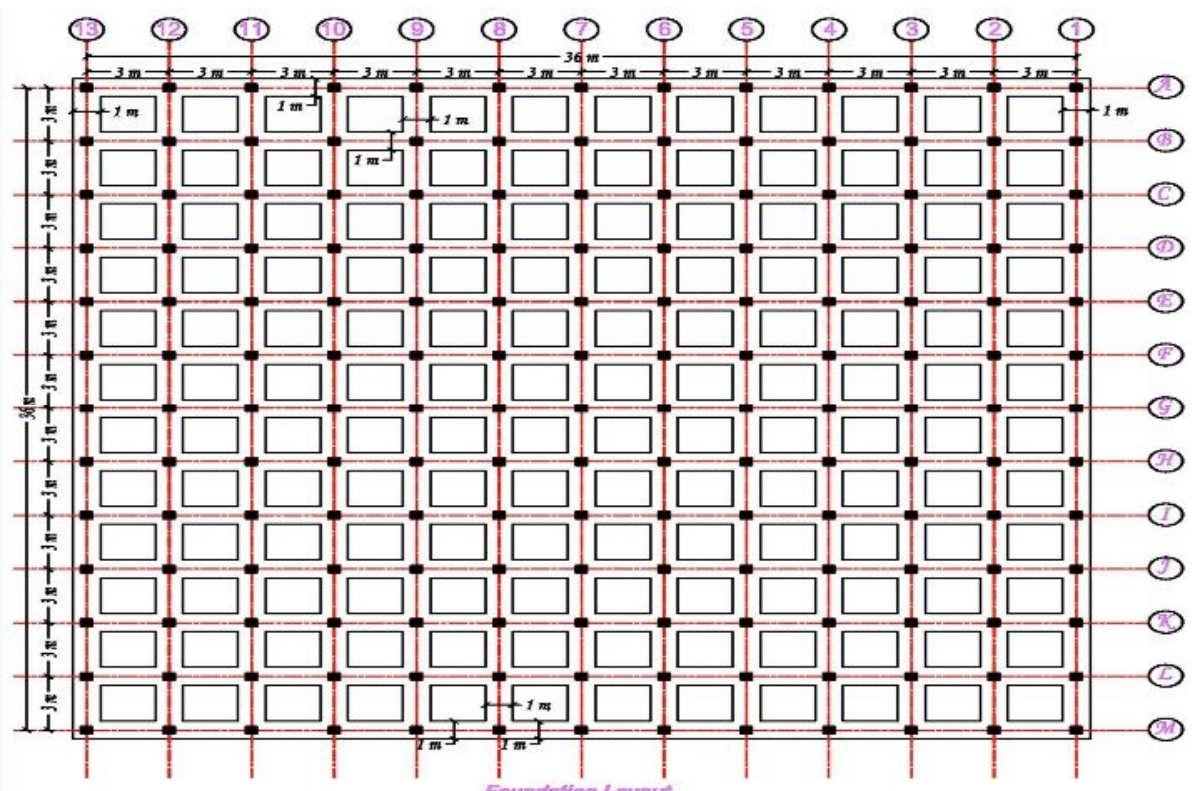

شكل (9) : مخطط الاسس في حالة التباعد بين الاعمدة 3.0 m X 3.0 .

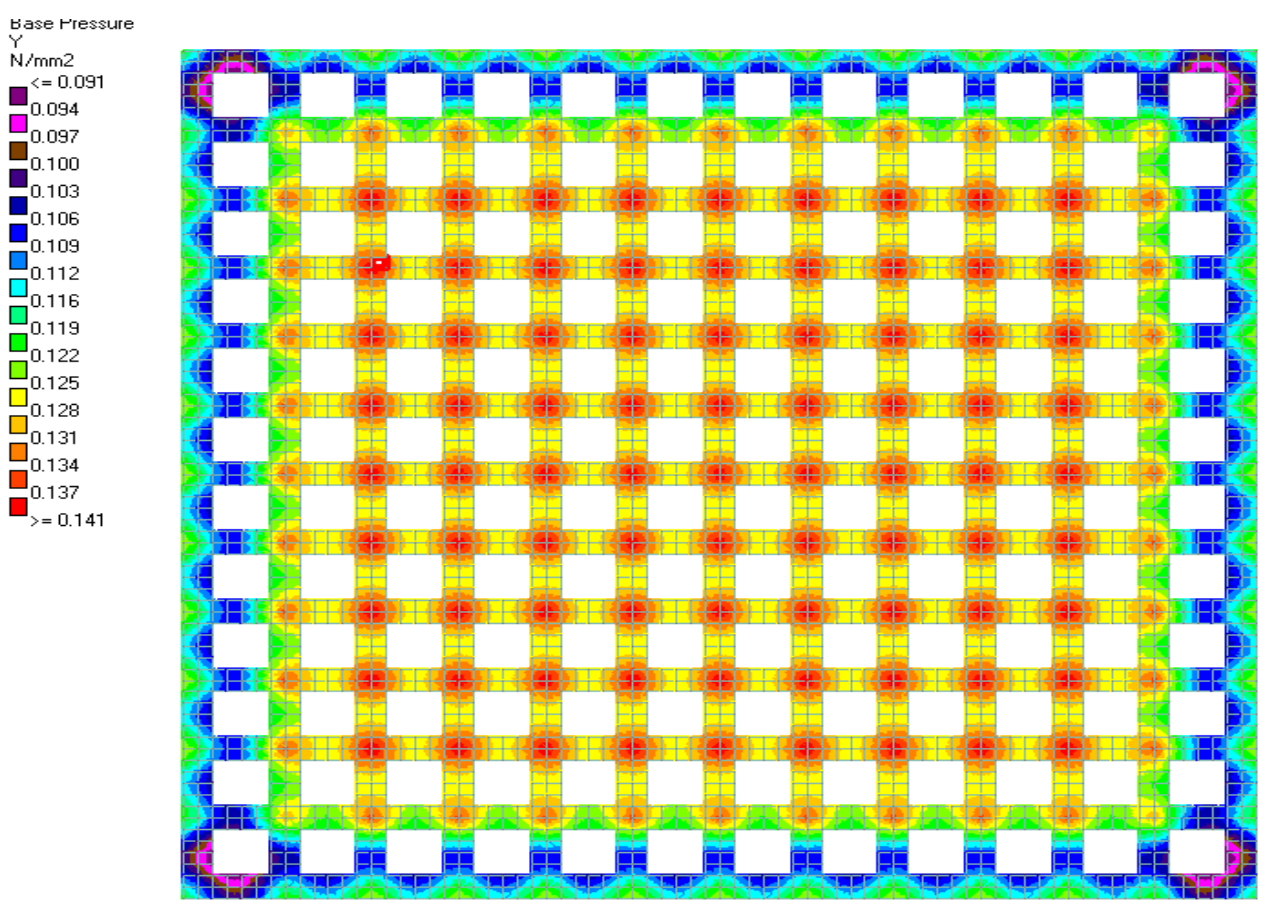

شكل (10) : مخطط يبين الضغط تحت الاسس في حالة التباعد بين الاعمدة 3.0 m X 3.0 . 


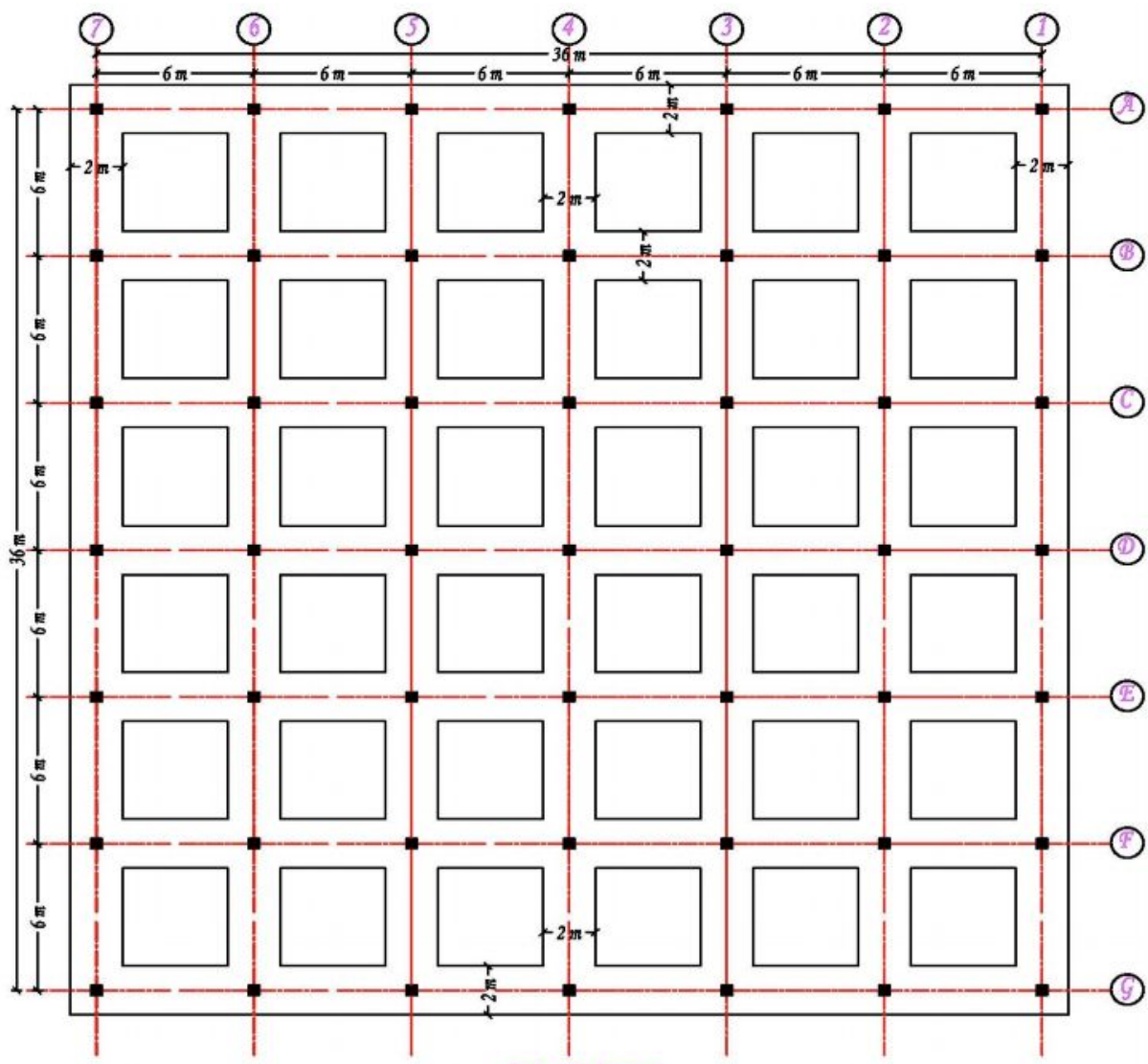

شكل (11) : مخطط الاسس في حالة التباعد بين الاعمدة 6.0 m X 6.0 .

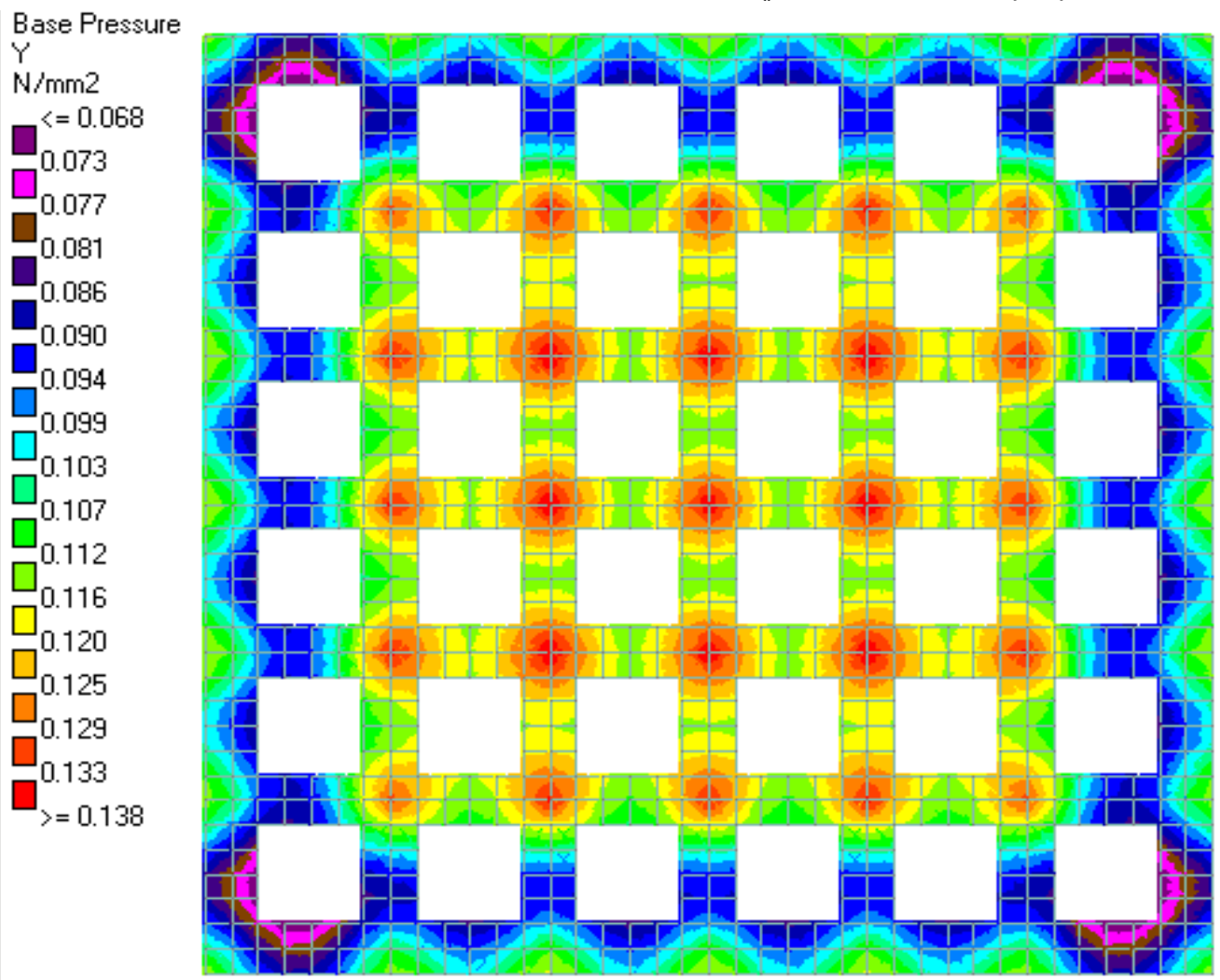

شكل (12) : مخطط يبين الضغط تحت الاسس في حالة التباعد بين الاعمدة 6.0 m X 6.0 . 
سعيد: تحديد نسبة تأثير أبعاد الفضاءات على التصميم الانثائي للابنية متعددة الطوابق

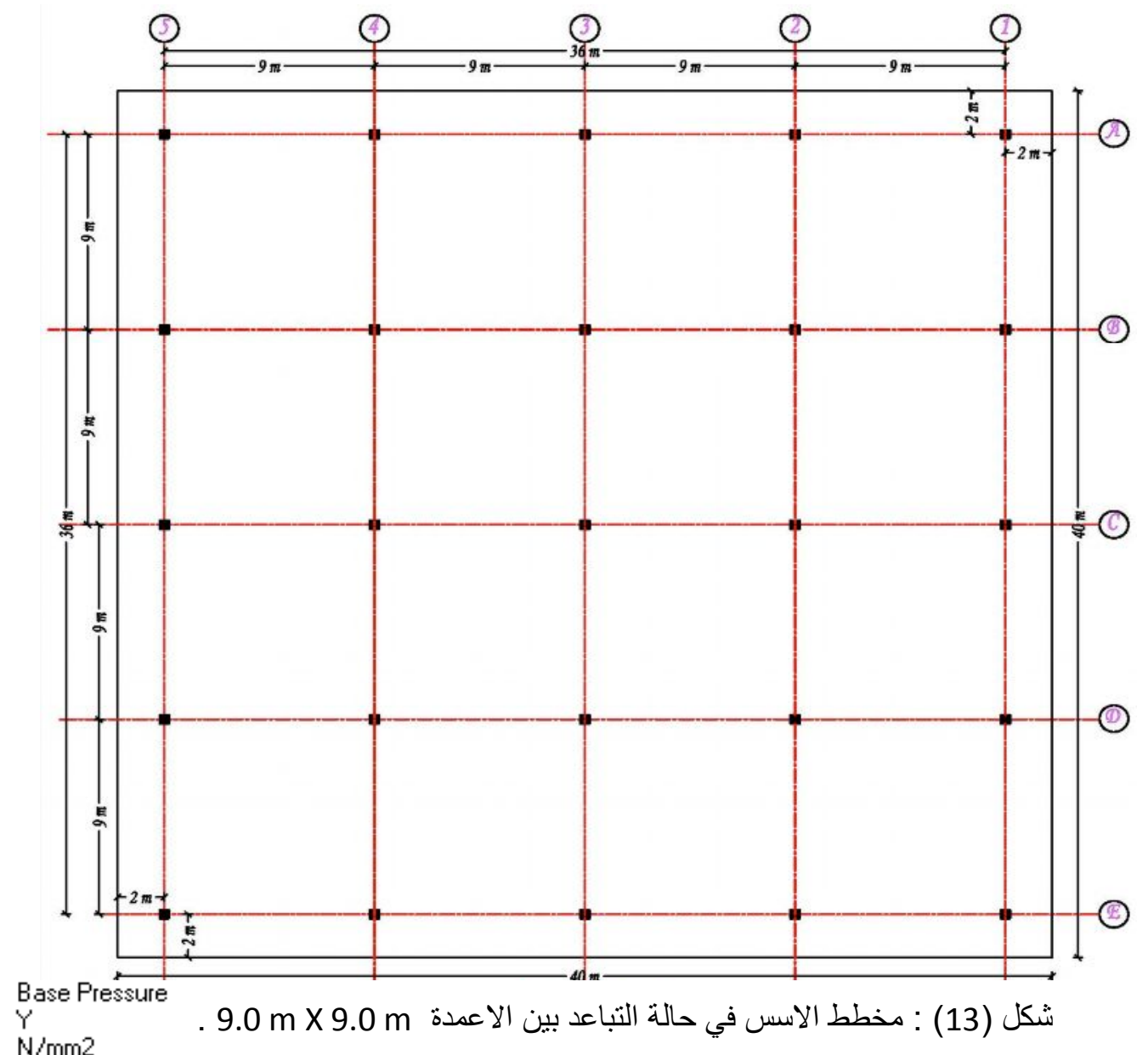

$\mathrm{N} / \mathrm{mm} 2$

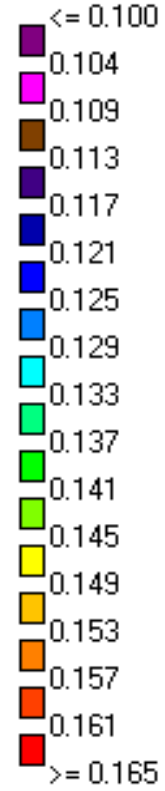

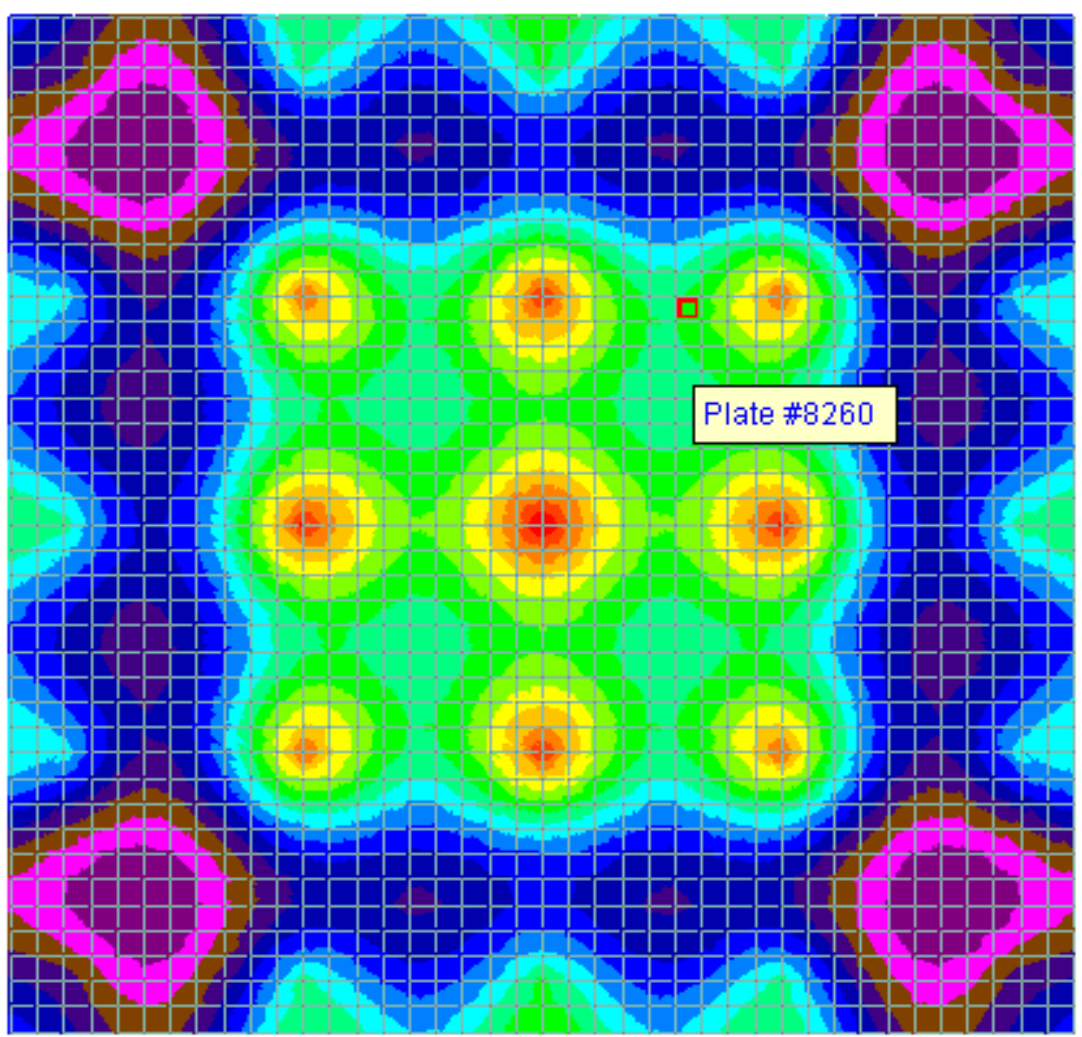

شكل (14) : مخطط يبين الضغط تحت الاسس في حالة التباعد بين الاعمدة 9.0 m X 9.0 . 
No.4

August 2011

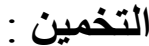

تبين الجداول (17 الى 35 ) نتائج عملية أحتساب الكميات للاحتمالات الثناثة :

جدول (17) حجم الخرسانة للسقوف

\begin{tabular}{|c|c|c|c|}
\hline الفضاء & m الابعاد & m السمك & الحجم m3 \\
\hline $3 * 3$ & $(36 * 36) * 4$ & 0.1 & 518.4 \\
\hline $6 * 6$ & $(36 * 36) * 4$ & 0.15 & 777.6 \\
\hline $9 * 9$ & $(36 * 36) * 4$ & 0.125 & 648 \\
\hline
\end{tabular}

جدول (18) حجم الخرسانة للاعتاب

\begin{tabular}{|c|c|c|c|c|}
\hline الفضاء & الابعاد m & الطول m & 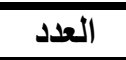 & الحجم m3 \\
\hline \multirow[t]{3}{*}{$3 * 3$} & $0.25 * 0.25$ & 36 & $13 * 4$ & 117 \\
\hline & $0.25 * 0.25$ & 32.75 & $13 * 4$ & 106.4 \\
\hline & & & & 233.4 \\
\hline \multirow[t]{3}{*}{$6 * 6$} & $0.35 * 0.35$ & 36 & $7 * 4$ & 123.5 \\
\hline & $0.35 * 0.35$ & 33.55 & $7 * 4$ & 115 \\
\hline & & & & 238.5 \\
\hline \multirow[t]{4}{*}{$9 * 9$} & $0.875 * 0.35$ & 36 & $5 * 4$ & 220.5 \\
\hline & $0.875 * 0.35$ & 34.25 & $5 * 4$ & 210 \\
\hline & $0.625 * 0.35$ & 34.25 & $8 * 4$ & 240 \\
\hline & & & & 670.5 \\
\hline
\end{tabular}

جدول (19) حجم الخرسانة للاعمدة

\begin{tabular}{|c|c|c|c|c|}
\hline الفضاء & الابعاد m & الطول m & العدد & الحجم m3 \\
\hline $3 * 3$ & $0.4 * 0.4$ & 3.0 & 169 & 81.12 \\
\hline & $0.35 * 0.35$ & 3.0 & 169 & 62.11 \\
\hline & $0.3 * 0.3$ & 3.0 & 169 & 45.63 \\
\hline & $0.25 * 0.25$ & 3.0 & 169 & 31.68 \\
\hline & & & & 220.55 \\
\hline $6 * 6$ & $0.5 * 0.5$ & 3.0 & $49 * 4$ & 147 \\
\hline $9 * 9$ & $0.6 * 0.6$ & 3.0 & $25 * 4$ & 108 \\
\hline
\end{tabular}

جدول (20) حجم الخرسانة للاسس

\begin{tabular}{|c|c|c|c|}
\hline & $\mathbf{m}$ الابع| & $\mathbf{m}$ & $\mathbf{m 3}$ اللحمث| \\
\hline $3 * 3$ & $37 * 37$ & 0.4 & 547.6 \\
\hline & $12 * 12 * 4$ & 0.4 & $230.4-$ \\
\hline & & & 317.2 \\
\hline $6 * 6$ & $38 * 38$ & 0.55 & 794.2 \\
\hline & $6 * 6 * 16$ & 0.55 & $316.8-$ \\
\hline & & & 477.4 \\
\hline $9 * 9$ & $40 * 40$ & 1.2 & 1920 \\
\hline
\end{tabular}


سعيد: تحديد نسبة تأثير أبعاد الفضاعات على التصميم الانثائي للابنية متعددة الطوابق

\begin{tabular}{|c|c|}
\hline \multicolumn{2}{|c|}{ جدول (21) الحجم الكلي للخرسانة } \\
\hline $\mathbf{m}$ الحضاء & $\mathbf{m 3}$ \\
\hline $3 * 3$ & 1289.55 \\
\hline $6 * 6$ & $\mathbf{1 6 4 0 . 5}$ \\
\hline $9 * 9$ & 3346.5 \\
\hline
\end{tabular}

جدول (22) حجم حديد تسليح السقوف لفضاء (3 × 3) مثر

\begin{tabular}{|c|c|c|c|}
\hline الطابق & حجم التسليح لفضاء & عدد الفضاءات & حجم حديد تسليح \\
\hline كل الطو ابق & $7488 * 10^{-6}$ & 144 & 4.313088 \\
\hline & & & $4.313 \mathrm{~m} 3$ \\
\hline
\end{tabular}

جدول (23) حجم حديد تسليح السقوف لفضاء (6 × 6) مثر

\begin{tabular}{|c|c|c|c|}
\hline الطابق & حجم التسليح لفضاء & عدد الفضـاءات & حجم حديد تسليح \\
\hline السطح & 0.03254 & 36 & 1.1715 \\
\hline الطو ابق & 0.03977 & $36 * 3$ & 4.2958 \\
\hline & & & $5.467 \mathrm{~m}^{3}$ \\
\hline
\end{tabular}

جدول (24) حجم تسليح السقوف لفضاء (9×9 (9) متر

\begin{tabular}{|c|c|c|c|c|}
\hline الطابق & حجم التسليح بالاتجاه الطويل & بالاتجاه القصبير & 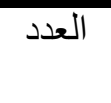 & حجم التسليح الكلي \\
\hline السطح & 0.01356 & 0.01898 & 48 & 1.5621 \\
\hline الطو ابق & 0.01356 & 0.033448 & $48 * 3$ & 6.76915 \\
\hline & & & & 8.3312 \\
\hline
\end{tabular}

جدول (25) حجم حديد التسليح لاعتاب فضاء (3×3) متر

\begin{tabular}{|c|c|c|c|c|}
\hline الموقع & الطابق & لعتب واحد التسليح & عدد العتبات & الحجم الكلي لحديد التسليح m3 \\
\hline B1 & كل الطوابق & $2034 * 10^{-6}$ & $48 * 4$ & $390528 * 10^{-6}$ \\
\hline B2 & كل الطو ابق & $2034 * 10^{-6}$ & $264 * 4$ & $2147904 * 10^{-6}$ \\
\hline حلقات & كل الطوابق & $2047 * 10^{-6}$ & $312 * 4$ & $2555280 * 10^{-6}$ \\
\hline & & & & 5.093 \\
\hline
\end{tabular}

\begin{tabular}{|c|c|c|c|c|}
\hline الموقع & الطابق & العدد & 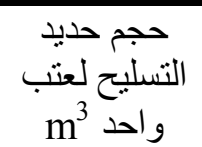 & الحجم الكلي لحديد \\
\hline $\mathrm{B} 2$ & باقي الطو ابق & $60 * 3$ & 0.013188 & 2.3738 \\
\hline $\mathrm{B} 1$ & باقي الطوابق & $24 * 3$ & 0.00844 & 0.60782 \\
\hline B2 & السطح & 60 & 0.009648 & 0.5788 \\
\hline B1 & السطح & 24 & 0.005424 & 0.13017 \\
\hline حلقات & كل الطو ابق & 336 & 0.0028704 & 0.96445 \\
\hline & & & & 4.655 \\
\hline
\end{tabular}




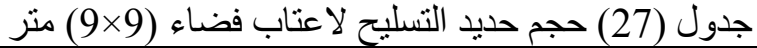

\begin{tabular}{|c|c|c|c|c|}
\hline الموقع & الطابق & حجم التسليح لعتب واحد m³ & عدد العتبات & الحجم الكلي لحديد التسليح m3 \\
\hline $\mathrm{B} 1$ & كل الطو ابق & $21666 * 10^{-6}$ & $8 * 3$ & $519984 * 10^{-6}$ \\
\hline $\mathrm{B} 2$ & كل الطو ابق & $27930 * 10^{-6}$ & $8 * 3$ & $670320 * 10^{-6}$ \\
\hline B3 & كل الطو ابق & $42630 * 10^{-6}$ & $12 * 3$ & $1534680 * 10^{-6}$ \\
\hline B4 & كل الطو ابق & $20724 * 10^{-6}$ & $12 * 3$ & $746064 * 10^{-6}$ \\
\hline B5 & كل الطو ابق & $21666 * 10^{-6}$ & $8 * 3$ & $519984 * 10^{-6}$ \\
\hline B1 & سطح & $14130 * 10^{-6}$ & 8 & $113040 * 10^{-6}$ \\
\hline $\mathrm{B} 2$ & سطح & $14472 * 10^{-6}$ & 8 & $115776 * 10^{-6}$ \\
\hline B3 & سطح & $33810 * 10^{-6}$ & 12 & $405720 * 10^{-6}$ \\
\hline B4 & سطح & $21666 * 10^{-6}$ & 12 & $259992 * 10^{-6}$ \\
\hline B5 & سطح & $18840 * 10^{-6}$ & 8 & $150720 * 10^{-6}$ \\
\hline حلقات & كل العتبات & $6770.4 * 10^{-6}$ & 192 & $1299917 * 10^{-6}$ \\
\hline اضافة & كل العتبات & $7236 * 10^{-6}$ & 160 & $115776 * 10^{-6}$ \\
\hline & & & 6.678 & 6.451 \\
\hline
\end{tabular}

جدول (28) حجم حديد التسليح الاعمدة لفضاء (3 × 3) متر

\begin{tabular}{|c|c|c|c|c|c|}
\hline رمز العمود & الطابق & الطول & العدد & مساحة التسليح & حجم حديد تسليح \\
\hline $\mathrm{C}_{1}$ & الثالث & 3.35 & 4 & $804 * 10^{-6}$ & $10774 * 10^{-6}$ \\
\hline $\mathrm{C}_{2}$ & الثالث & 3.35 & 46 & $804^{*} 10^{-6}$ & $123896 * 10^{-6}$ \\
\hline$\overline{C_{3}}$ & الثالث & 3.35 & 119 & $804 * 10^{-6}$ & $320515 * 10^{-6}$ \\
\hline$\overline{\mathrm{C}_{1}}$ & الثاني & 3.85 & 4 & $1016^{*} 10^{-6}$ & $15646^{*} 10^{-6}$ \\
\hline$\overline{\mathrm{C}_{2}}$ & الثاني & 3.85 & 46 & $1016^{*} 10^{-6}$ & $179934 * 10^{-6}$ \\
\hline $\mathrm{C}_{3}$ & الثاني & 3.85 & 119 & $1016^{*} 10^{-6}$ & $465480 * 10^{-6}$ \\
\hline $\mathrm{C}_{1}$ & الاول & 3.85 & 4 & $1256^{*} 10^{-6}$ & $19342 * 10^{-6}$ \\
\hline $\mathrm{C}_{2}$ & الاول & 3.85 & 46 & $1256^{*} 10^{-6}$ & $222438 * 10^{-6}$ \\
\hline $\mathrm{C}_{3}$ & الاول & 3.85 & 119 & $1256^{*} 10^{-6}$ & $575436^{*} 10^{-}$ \\
\hline$\overline{\mathrm{C}_{1}}$ & الارضي & 4.55 & 4 & $1960 * 10^{-6}$ & $35672 * 10^{-6}$ \\
\hline $\mathrm{C}_{2}$ & الارضي & 4.55 & 46 & $1960 * 10^{-6}$ & $410228 * 10^{-6}$ \\
\hline $\mathrm{C}_{3}$ & الارضي & 4.55 & 119 & $1960 * 10^{-6}$ & $1061242 * 10^{-6}$ \\
\hline & & & & & $3.44 \mathrm{~m}^{3}$ \\
\hline
\end{tabular}

جدول (29) حجم حديد التسليح لرباطات الاعمدة لفضاء (3×3) متر

\begin{tabular}{|c|c|c|c|c|}
\hline الطابق & طول الحلقة م & عدد الحلقات & مساحة القضيب م3 & الحجم الكلي للحلقات \\
\hline الارضي & 1.5 & $169 * 8$ & $78^{*} 10^{-6}$ & 0.158184 \\
\hline الاول & 1.3 & $169 * 12$ & $78 * 10^{-6}$ & 0.205639 \\
\hline الثاني & 1.1 & $169 * 14$ & $78^{*} 10^{-6}$ & 0.203003 \\
\hline الثالث & 0.9 & $169 * 14$ & $78^{*} 10^{-6}$ & 0.166093 \\
\hline & & & & $0.733 \mathrm{~m}^{3}$ \\
\hline
\end{tabular}


سعيد: تحديا نسبة تأثير أبعاد الفضاءات على التصميم الانشائي للابنية متعددة الطوابق

جدول (30) حجم حديد التسليح لاعمدة فضاء (6 × 6) منر

\begin{tabular}{|c|c|c|c|c|c|}
\hline رمز العمود & الطابق & العدد & الطول & مساحة التسليح & حجم حديد تسليح \\
\hline $\mathrm{C}_{1}$ & كل الطو ابق & $4 * 3$ & 3.5 & 0.003216 & 0.135072 \\
\hline $\mathrm{C}_{2}$ & كل الطو ابق & $20 * 3$ & 3.5 & 0.002512 & 0.67536 \\
\hline $\mathrm{C}_{3}$ & كل الطو ابق & $25 * 3$ & 3.5 & 0.003216 & 0.8442 \\
\hline $\mathrm{C}_{1}$ & الارضي & 4 & 4.8 & 0.002512 & 0.04823 \\
\hline $\mathrm{C}_{2}$ & الارضي & 20 & 4.8 & 0.002512 & 0.241152 \\
\hline $\mathrm{C}_{3}$ & الارضي & 25 & 4.8 & 0.002512 & 0.30144 \\
\hline & & & & & $2.098 \mathrm{~m}^{3}$ \\
\hline
\end{tabular}

جدول (31) حجم حديد التسليح لرباطات الاعمدة لفضاء (6)6ماتر

\begin{tabular}{|c|c|c|c|c|}
\hline كل الطو ابق & طول الحلقة & العدد & مساحة التسليح & الحجم الكلي للحلقات \\
\hline كل الطو ابق & 2.0 & $49 * 4 * 12$ & $78 * 10^{-6}$ & 0.367 \\
\hline
\end{tabular}

جدول (32) حجم حديد تسليح للاعمدة لفضاء (9×9)متر

\begin{tabular}{|c|c|c|c|c|c|}
\hline رمز العمود & الطابق & الطول & العدد & مساحة حديد التسليح & حجم حديد تسليح لعمود \\
\hline $\mathrm{C}_{1}$ & الارضي & 6 & 4 & $7840 * 10^{-6}$ & $18816 * 10^{-6}$ \\
\hline $\mathrm{C}_{1}$ & باقي الطوابق & 4 & 12 & $7840 * 10^{-6}$ & $376320 * 10^{-6}$ \\
\hline $\mathrm{C}_{4}$ & الارضي & 6 & 9 & $12300 * 10^{-6}$ & $664200 * 10^{-6}$ \\
\hline $\mathrm{C}_{4}$ & الاول & 4 & 9 & $9840 * 10^{-6}$ & $354240 * 10^{-6}$ \\
\hline $\mathrm{C}_{4}$ & الثاني & 4 & 9 & $3768 * 10^{-6}$ & $135648 * 10^{-6}$ \\
\hline $\mathrm{C}_{4}$ & الثالث & 4 & 9 & $3768 * 10^{-6}$ & $135648 * 10^{-6}$ \\
\hline $\mathrm{C}_{2}$ & الارضي & 6 & 6 & $12300 * 10^{-6}$ & $442800 * 10^{-6}$ \\
\hline $\mathrm{C}_{2}$ & باقي الطو ابق & 4 & 18 & $12300 * 10^{-6}$ & $885600 * 10^{-6}$ \\
\hline $\mathrm{C}_{3}$ & ارضي & 6 & 6 & $5024 * 10^{-6}$ & $180864^{*} 10^{-6}$ \\
\hline $\mathrm{C}_{3}$ & باقي الطو ابق & 4 & 18 & $5024 * 10^{-6}$ & $361728 * 10^{-6}$ \\
\hline حلقات & كل الطوابق & 6 & $100 * 9$ & $78 * 10^{-6}$ & $421200 * 10^{-6}$ \\
\hline & & & & & $3.8 \mathrm{~m}^{3}$ \\
\hline
\end{tabular}

جدول (33) حجم حديد التسليح الاساس لفضاء (3×3)م

\begin{tabular}{|c|c|c|c|}
\hline شكل التسليح & حجم التسليح للاساس & عدد الاسس & الحجم الكلي لتسليح \\
\hline طولي & 0.089244 & $13 * 2$ & 2.320344 \\
\hline حلقات & 0.0279792 & $13 * 2$ & 0.7274592 \\
\hline & & & 3.0478032 \\
\hline
\end{tabular}

جدول (34) حجم حديد التسليح الاساس لفضاء (6×6)م

\begin{tabular}{|c|c|c|c|}
\hline شكل التسليح & حجم التسليح للاساس & عدد الاسس & الحجم الكلي لتسليح \\
\hline طولي & 0.334096 & $7 * 2$ & 4.677344 \\
\hline حلقات & 0.150720 & $7 * 2$ & 2.110080 \\
\hline & & & 6.787424 \\
\hline
\end{tabular}


جدول (35) حجم حديد التسليح الاساس لفضاء (9×9)م

\begin{tabular}{|c|c|c|c|c|}
\hline الموقع & طول القضيب & 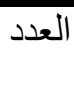 & لقضيب واحة التسليح م2 & الحجم الكلي لتسليح \\
\hline اعلى & 40 & 267 & $615 * 10^{-6}$ & $6568200 * 2=13136400$ \\
\hline اسفل & 40 & 267 & $615 * 10^{-6}$ & $6568200 * 2=13136400$ \\
\hline الوسط & 40 & 134 & $615 * 10^{-6}$ & $3296400 * 2=6592800$ \\
\hline & & & & $32.865 \mathrm{~m}^{3}$ \\
\hline
\end{tabular}

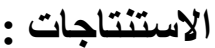

يبين الجدول رقم (36) كمية الخرسانة لكل من السقوف و العتبات و الاعمدة و الاسس و المجمو ع الكلي للكميات

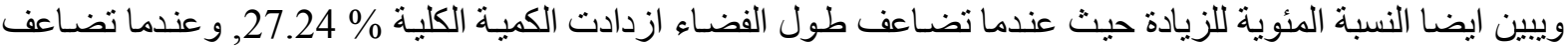

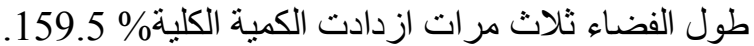

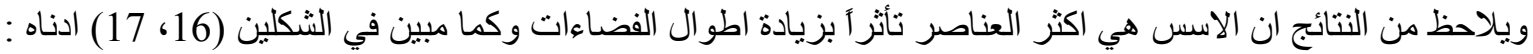
جدول رقم (36) ملخص لكمية الخرسانة مع طول الفضاء

\begin{tabular}{|c|c|c|c|c|c|c|}
\hline \multicolumn{6}{|c|}{ كمية الخرسانة (مُ2/2) } & \multirow[b]{2}{*}{ الفضاء (م) } \\
\hline النسبة المئوية للزيادة & المجموع الكلي & الأسس & الأعمدة & العتبات & السقوف & \\
\hline $0 \%$ & 0.995 & 0.2448 & 0.17 & 0.1723 & 0.4 & $3 \mathrm{~m}$ \\
\hline $27.24 \%$ & 1.266 & 0.3684 & 0.1134 & 0.184 & 0.6 & $6 \mathrm{~m}$ \\
\hline $159.5 \%$ & 2.582 & 1.481 & 0.0833 & 0.5174 & 0.5 & $9 \mathrm{~m}$ \\
\hline
\end{tabular}

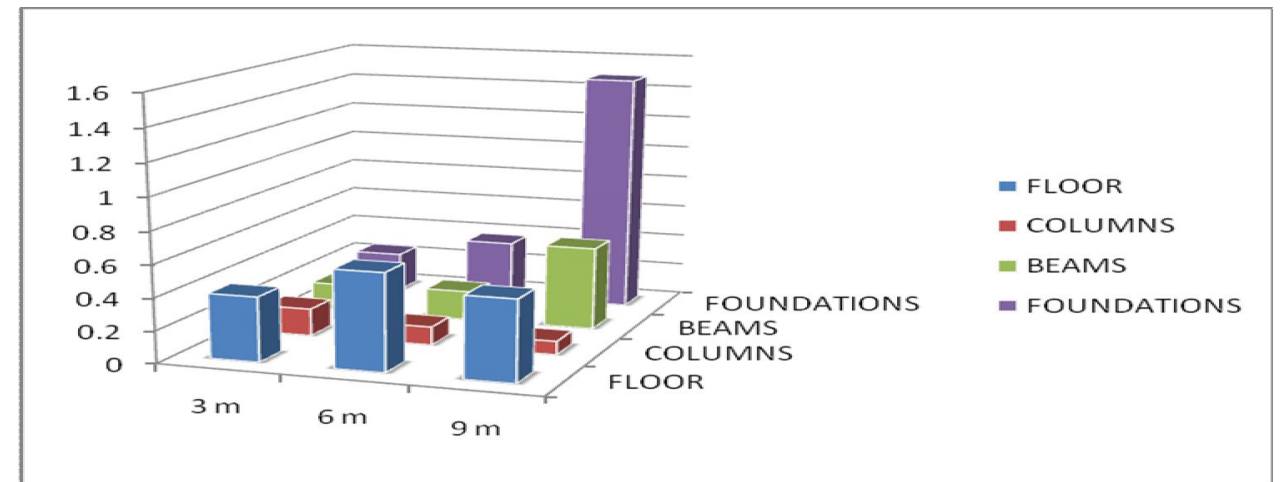

شكل (16) : مخطط يبين نسبة نأثير طول الفضاء على كمية الخرسانة لمختلف الاعضاء الانشائية .

ويبين الجدول رقم (37) وزن حديد التسليح اللازم لكل من متر مربع من البناية بالنسبة للسقوف و العتبات

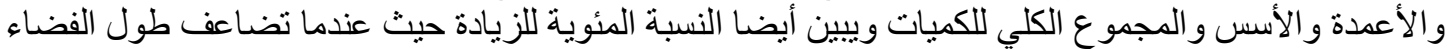

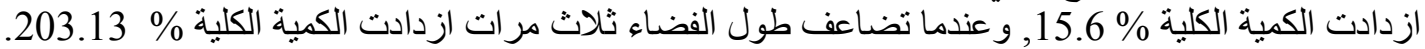
جدول رقم (37) ملخص لوزن حديد التسليح* مع طول الفضاء

\begin{tabular}{|c|c|c|c|c|c|c|}
\hline \multicolumn{6}{|c|}{ وزن حديد التسليح (كغم/م²) } & \multirow{2}{*}{ الفضاء (م) } \\
\hline النسبة المئوية للزيادة & المجموع الكلي & الأسس & الأعمدة & العتبات & السقوف & \\
\hline $0 \%$ & 101.40 & 20.13 & 24.32 & 30.85 & 26.1 & $3 \mathrm{~m}$ \\
\hline $15.4 \%$ & 117 & 41.11 & 14.93 & 28.2 & 32.76 & $6 \mathrm{~m}$ \\
\hline $202.6 \%$ & 306.8 & 199.07 & 26.11 & 45.39 & 40.58 & $9 \mathrm{~m}$ \\
\hline
\end{tabular}




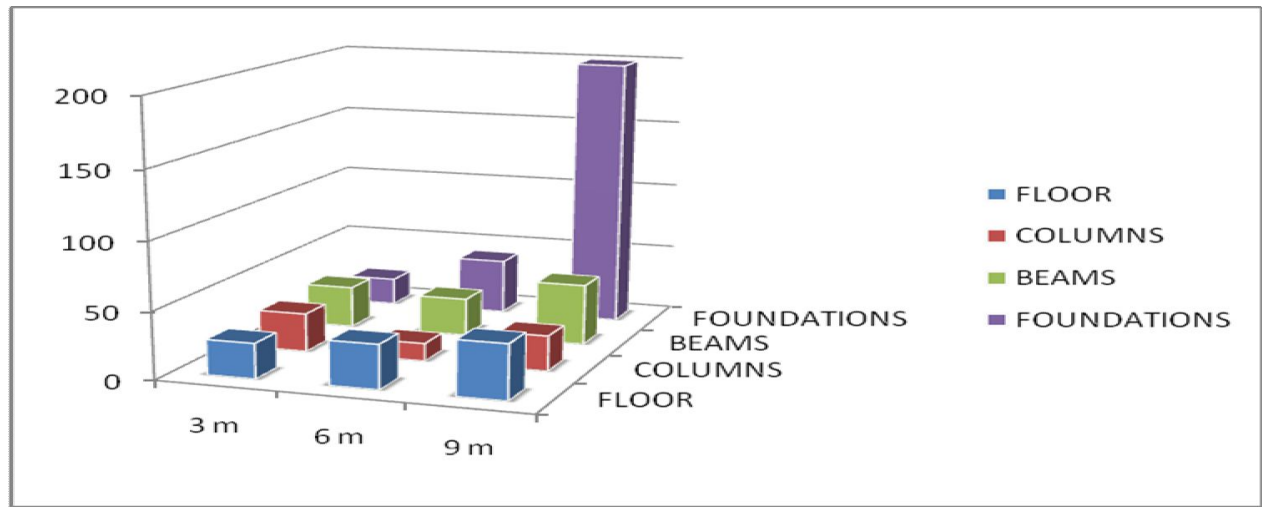

شكل (17) : مخطط ييين نسبة تأثير طول الفضاء على كمية حديد التسليح لمختلف الاعضاء الانشائية .

تبين الاشكال (18-25) نوع العلاقة مابين طول الفضاء وحجم الخرسانة وحجم حديد التسليح للاعضاء الانشائية للمبنى الآن

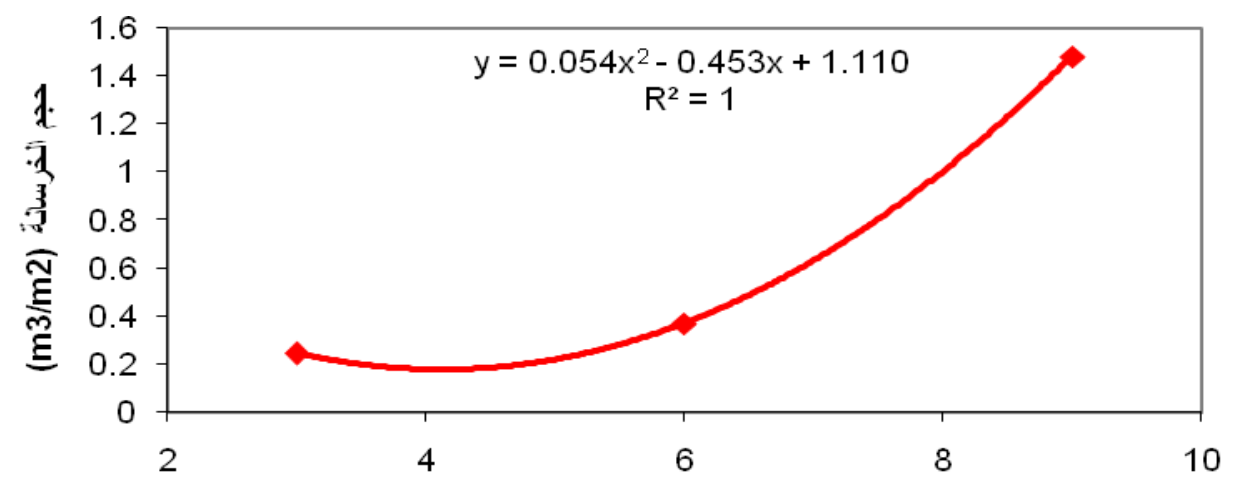

(m) طون الفضاء

شكل (18) : مخطط يبين العلاقة بين طول الفضاء وحجم الخرسانة للاسس .

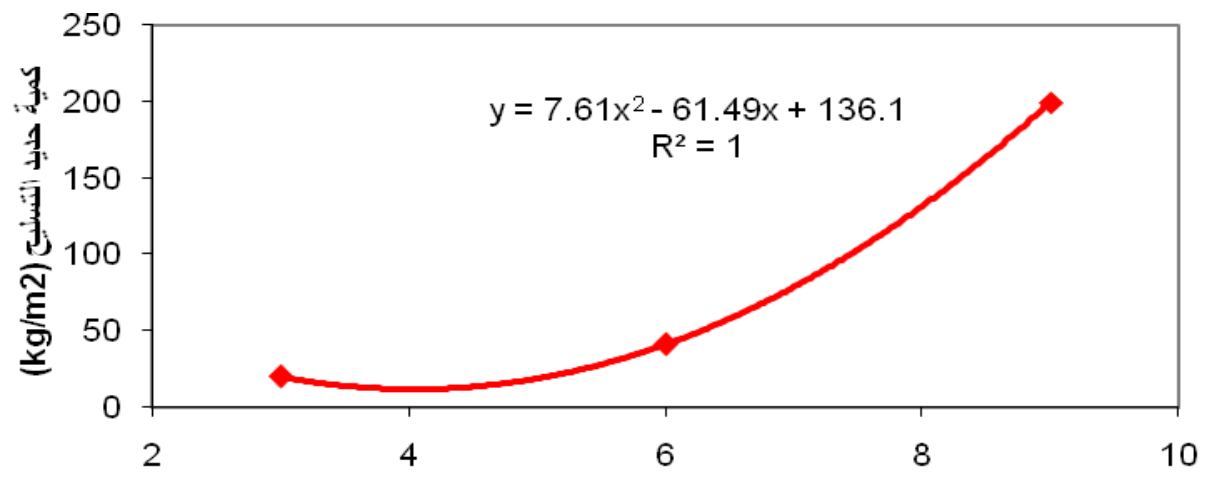

(m) طون الفضاء

شكل (19) : مخطط ييين العلاقة بين طول الفضاء وكمية حديد التسليح للاسس . 


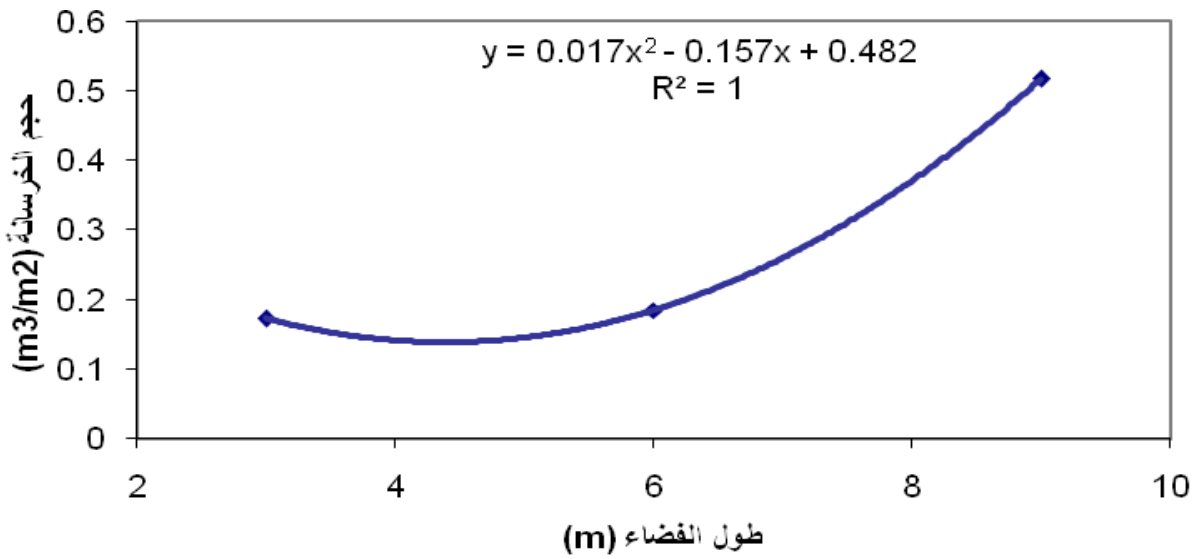

شكل (20) : مخطط يبين العلاقة بين طول الفضاء وحجم الخرسانة للاعتاب .

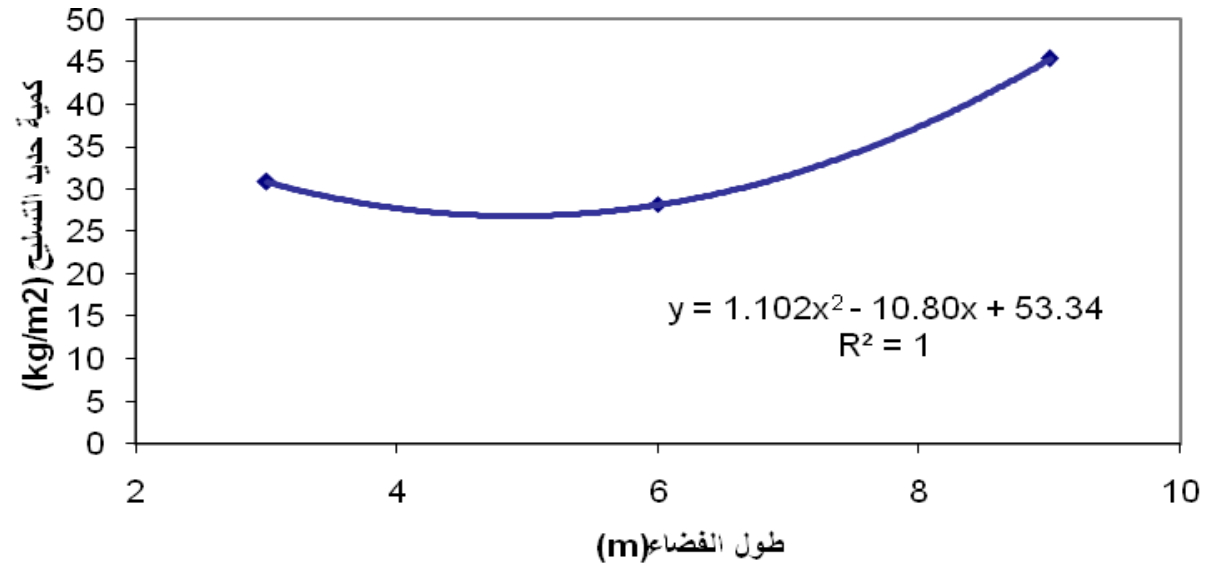

شكل (21) : مخطط ييين العلاقة بين طول الفضاء وكمية حديد التسليح للاعتاب .

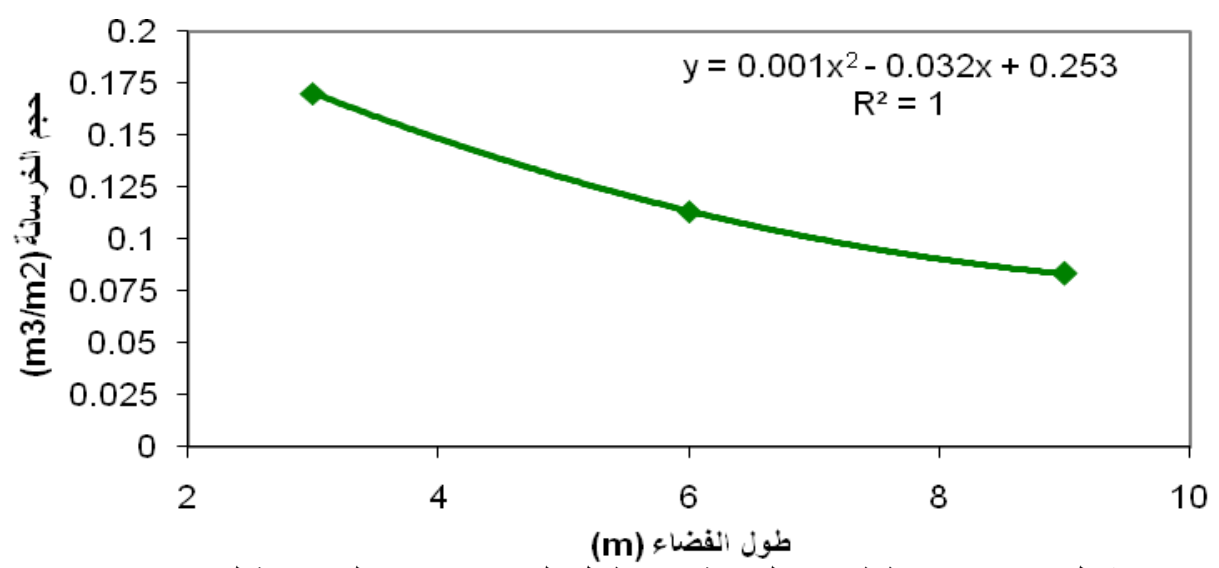

شكل (22) : مخطط ييين العلاقة بين طول الفضاء وحجم الخرسانة للاعمدة . 


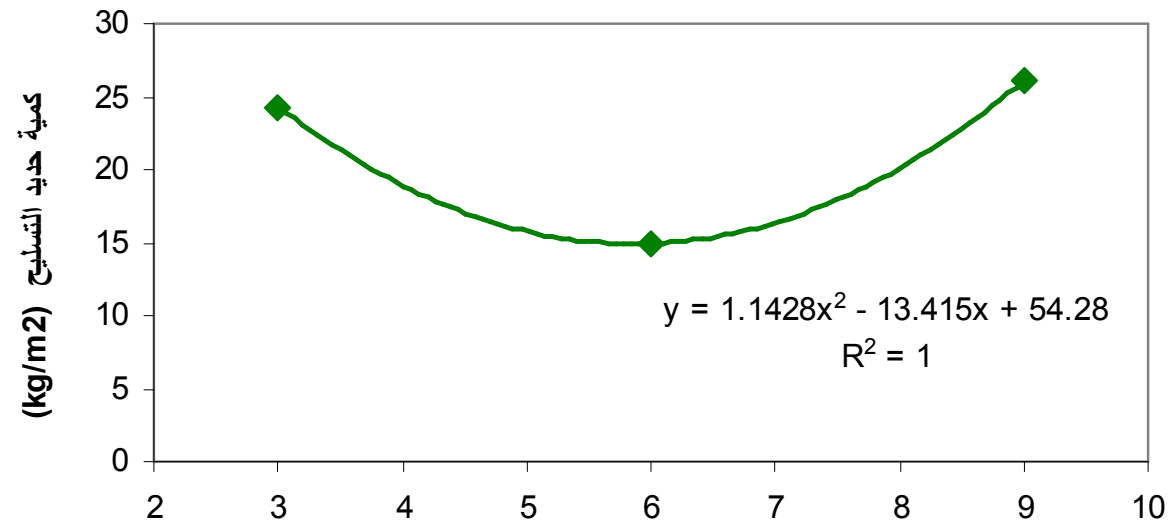

شكل (23) : مخطط ييين العلاقة بين طول الفضاء وكمية حديد التسليح للاعدة .

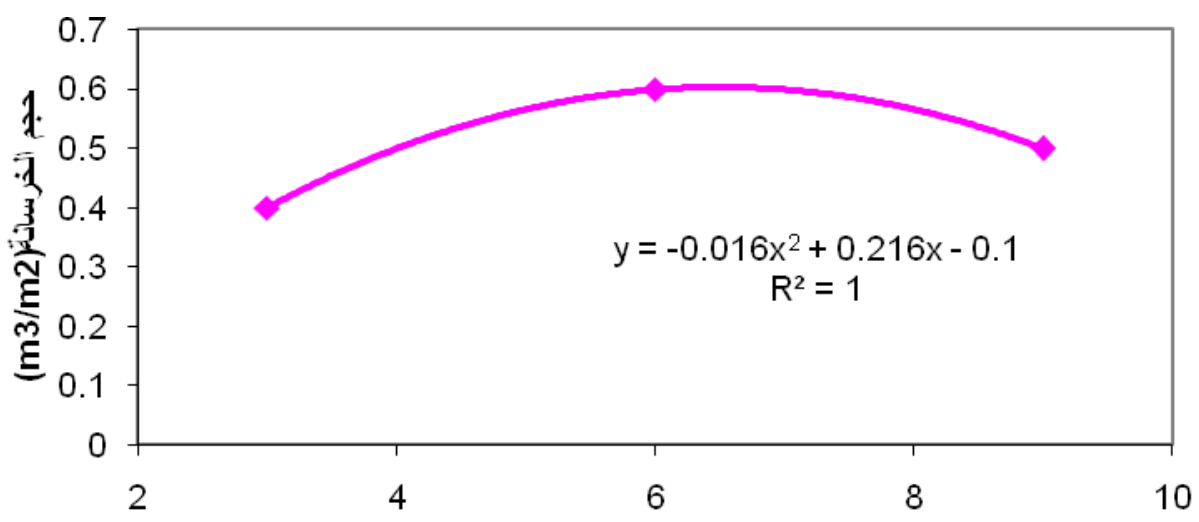

طول الفضاء (m)

شكل (24) : مخطط ييين العلاقة بين طول الفضاء وحجم الخرسانة للسقوف .

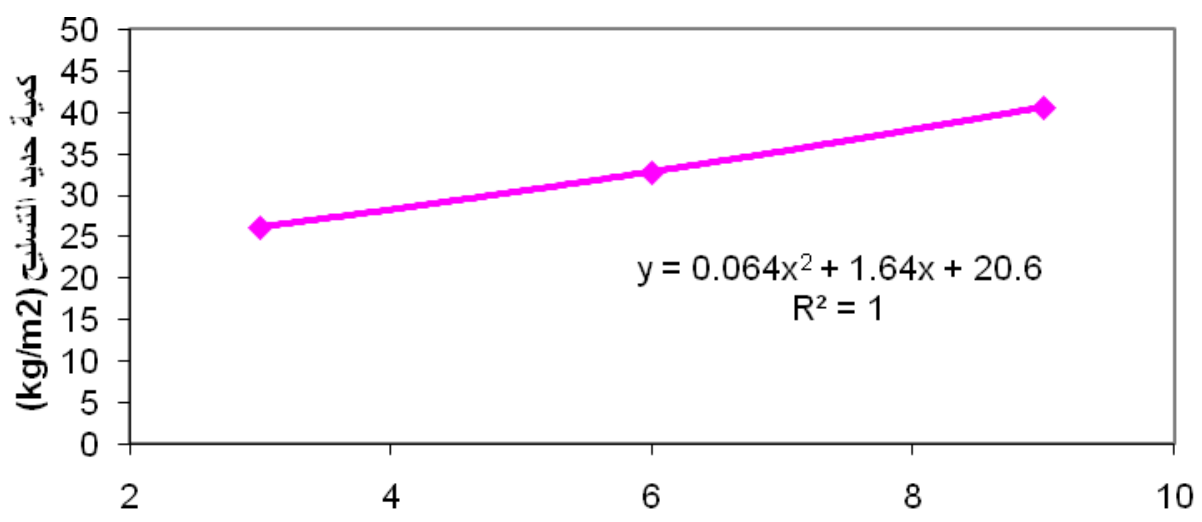

(m) طون الفضاء

شكل (25) : مخطط ييين العلاقة بين طول الفضاء وكمية حديد التسليح للسقوف . 
يبين الثكل رقم (26) علاقة طول الفضاء بالحجم الكلي للخرسانة,حيث نلاحظ بزيادة طول الفضاء يزداد حجم الخرسانة, $y=0.0581 x^{2}-0.4322 x+1.769$ و المعادلة التي تحكم هذه الزيادة هي : $y=0.0581 x^{2}-0.4322 x+1.769$ = y $\mathrm{Y}=0.965 \mathrm{~m}^{3} / \mathrm{m}^{2}$ و

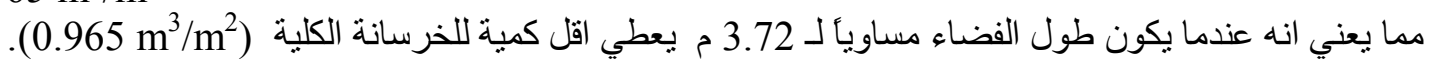

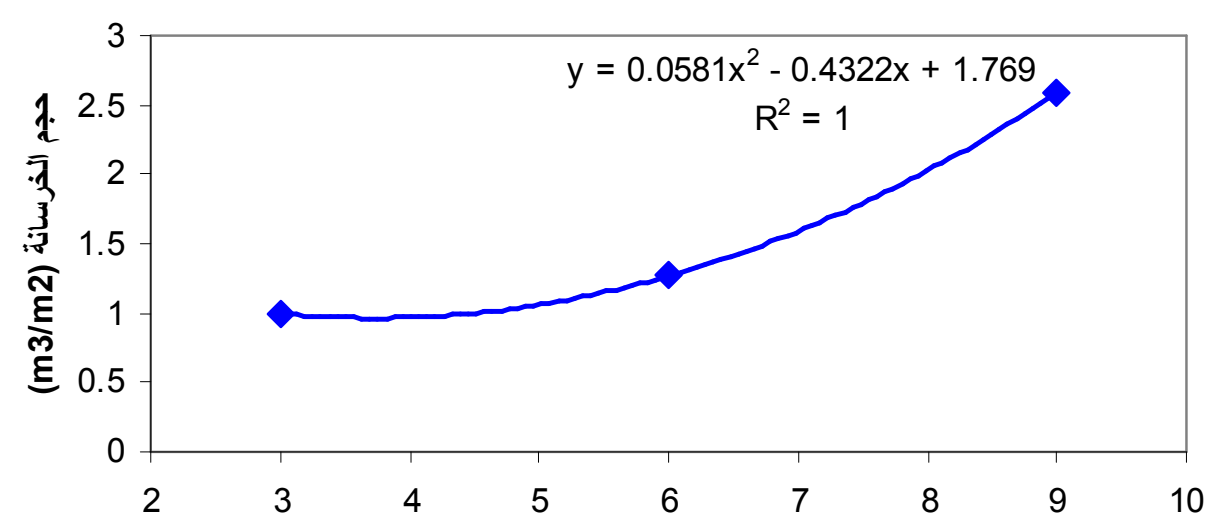

(m) طول الفضاء

\section{شكل (26) علاقة طول الفضاء بالحجم الكلي للخرسـانة}

ويبين الثكل رقم (27) علاقة طول الفضاء بالوزن الكلي لحديد التسليح ,حيث نلاحظ بزيادة طول الفضـاء يزداد الوزن, $y=9.6672 x^{2}-81.742 x+259.43$ و المعادلة التي تحكم هذه الزيادة هي: = وزن حديد التسليح (كغم/م²) Y (م) =X

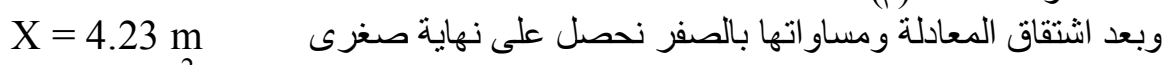
$\mathrm{Y}=86.64 \mathrm{~kg} / \mathrm{m}^{2}$

مما بعني انه عندما يكون طول الفضاء مساويأ لـ 4.23 م بعطي اقل كمية لحديد التسليح الكلية (20/m 86.64 ) . .

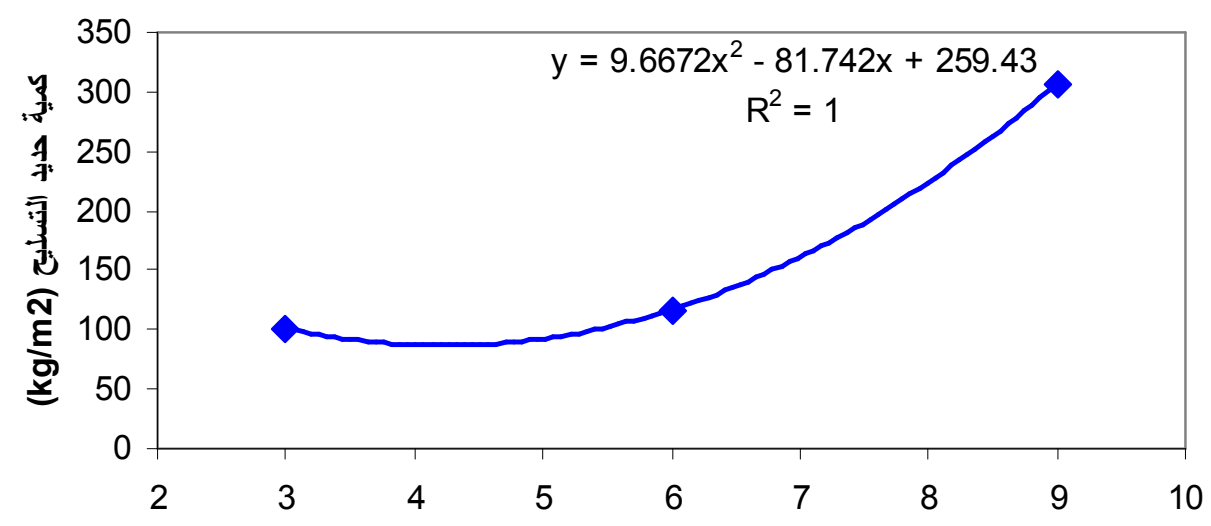

(m) طول الفضاء

شكل (27) علاقة طول الفضاء بكمية حليد التسليح الكليـة 


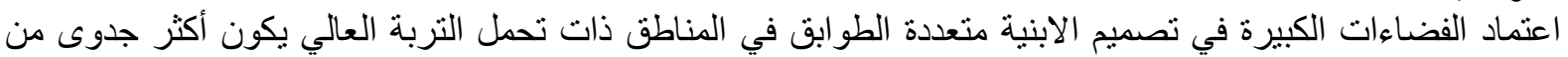

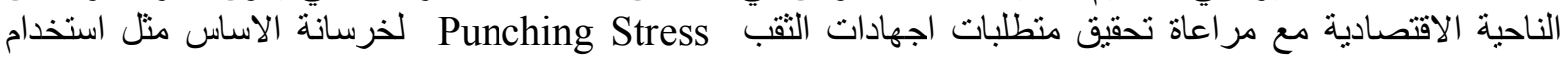

. Pedestals

\section{المصادر}

1. الطعان ، سعد علي ، "اساسيات الخرسانة المسلحة" ، دار الكتب للطباعة و النشر ، الموصل، 1989.

2. Winter, G. and Nilson, A.H.; "Design of Concrete Structures "; $10^{\text {th }}$ Edition, 1986, McGraw-Hill International editions.

3. STAAD PRO2007 software manual, http://www.reiworld.com.

4. ACI Committee 318-08; "Building Code Requirements for Structural Concrete and Commentary ", 2008.

5. Joseph E. Bowles, RE., S.E.; "Foundation analysis and design "; $5^{\text {th }}$ Edition, McGraw-Hill International editions, 1997. 